\title{
Doubly probabilistic representation for the stochastic porous media type equation
}

\author{
Viorel Barbu ${ }^{\mathrm{a}}$, Michael Röckner ${ }^{\mathrm{b}}$ and Francesco Russo ${ }^{\mathrm{c}}$ \\ ${ }^{a}$ University Al.I. Cuza, Ro-6600 Iasi, Romania \\ ${ }^{\mathrm{b}}$ Fakultät für Mathematik, Universität Bielefeld, D-33615 Bielefeld, Germany \\ ${ }^{\mathrm{c}}$ ENSTA ParisTech, Université Paris-Saclay, Unité de Mathématiques appliquées, 828, boulevard des Maréchaux, F-91120 Palaiseau, France
}

Received 15 May 2014; revised 4 August 2016; accepted 5 August 2016

\begin{abstract}
The purpose of the present paper consists in proposing and discussing a doubly probabilistic representation for a stochastic porous media equation in the whole space $\mathbb{R}^{1}$ perturbed by a multiplicative colored noise. For almost all random realizations $\omega$, one associates a stochastic differential equation in law with random coefficients, driven by an independent Brownian motion.
\end{abstract}

Résumé. Cet article propose et discute une représentation doublement probabiliste pour une équation des milieux poreux stochatique dans l'espace tout entier $\mathbb{R}^{1}$, perturbée par un bruit multiplicatif coloré. Pour presque toute réalisation $\omega$ de l'aléa, on associe une équation différentielle stochastique en loi avec coefficients aléatoires, dirigée par un mouvement brownien indépendant.

MSC: 35R60; 60H15; 60H30; 60H10; 60G46; 35C99; 58J65; 82C31

Keywords: Stochastic partial differential equations; Infinite volume; Singular porous media type equation; Doubly probabilistic representation; Multiplicative noise; Singular random Fokker-Planck type equation; Filtering

\section{Introduction}

We consider a function $\psi: \mathbb{R} \rightarrow \mathbb{R}$ and real functions $e^{0}, \ldots, e^{N}$ on $\mathbb{R}$, for some strictly positive integer $N$. In the whole paper, the following assumption will be in force.

\section{Assumption 1.1.}

- $|\psi(u)| \leq$ const $|u|, u \geq 0$. In particular, $\psi(0)=0$.

- $\psi: \mathbb{R} \rightarrow \mathbb{R}$ is a continuous function such that its restriction to $\mathbb{R}_{+}$is monotone increasing. Moreover we also suppose that $\lim _{u \rightarrow 0} \frac{\psi(u)}{u}$ exists.

- Let $e^{i} \in C_{\mathrm{b}}^{2}(\mathbb{R}), 0 \leq i \leq N$.

Let $T>0$ and $(\Omega, \mathcal{F}, P)$, be a fixed probability space. A generic element of $\Omega$ will be denoted by $\omega .\left(\mathcal{F}_{t}, t \in[0, T]\right)$ will stand for a filtration, fulfilling the usual conditions and we suppose $\mathcal{F}=\mathcal{F}_{T}$. Let $\mu(t, \xi), t \in[0, T], \xi \in \mathbb{R}$, be a random field of the type

$$
\mu(t, \xi)=\sum_{i=1}^{N} e^{i}(\xi) W_{t}^{i}+e^{0}(\xi) t, \quad t \in[0, T], \xi \in \mathbb{R}
$$


where $W^{i}, 1 \leq i \leq N$, are independent continuous $\left(\mathcal{F}_{t}\right)$-Brownian motions on $(\Omega, \mathcal{F}, P)$, which are fixed from now on until the end of the paper.

For technical reasons we will sometimes set $W_{t}^{0} \equiv t$. We focus on a stochastic partial differential equation of the following type:

$$
\left\{\begin{array}{l}
\partial_{t} X(t, \xi)=\frac{1}{2} \partial_{\xi \xi}^{2}(\psi(X(t, \xi)))+X(t, \xi) \partial_{t} \mu(t, \xi), \\
X(0, \mathrm{~d} \xi)=x_{0}(\mathrm{~d} \xi)
\end{array}\right.
$$

which holds in the sense of Definition 2.9, where $x_{0}$ is a given probability measure on $\mathbb{R}$. The stochastic multiplication above is of Itô type. We look for a solution of (1.1) with time evolution in $L^{1}(\mathbb{R})$. Since $\psi$ restricted to $\mathbb{R}_{+}$is nonnegative, Assumption 1.1 implies $\psi(u)=\Phi^{2}(u) u, u \geq 0, \Phi: \mathbb{R}_{+} \rightarrow \mathbb{R}$ being a non-negative continuous function which is bounded on $\mathbb{R}_{+}$.

\section{Remark 1.2.}

1. In the sequel we will consider, without further comments extensions of $\psi$ (and $\Phi$ ) to the real line which fulfill the first two items of Assumption 1.1 for $u \in \mathbb{R}$ instead of $u \geq 0$.

2. The restriction on $u \mapsto \Phi(u)$ introduced in Assumption 1.1 to be continuous is not always necessary, but here we assume this for simplicity.

When $\psi(u)=|u|^{m-1} u, m>1,(1.1)$ and $\mu \equiv 0,(1.1)$ is nothing else but the classical porous media equation. When $\psi$ is a general increasing function (and $\mu \equiv 0$ ), there are several contributions to the analytical study of (1.1), starting from [12] for existence, [15] for uniqueness in the case of bounded solutions and [13] for continuous dependence on the coefficients. Those are the classical references when the space variable varies on the real line. For equations in a bounded domain and Dirichlet boundary conditions, for simplicity, we only refer to monographs, e.g. [1,2,26,28].

As far as the stochastic porous media is concerned, most of the work for existence and uniqueness concerned the case of bounded domain, see for instance [3-5]. In the infinite volume case, i.e. when the underlying domain is $\mathbb{R}^{d}$, well-posedness was fully analyzed in [22], when $\psi$ is polynomially bounded (including the fast diffusion case) when the space dimension is $d \geq 3$. [8] established existence and uniqueness for any dimension $d \geq 1$ and the authors obtained estimates for finite time extinction. To the best of our knowledge, except for [22] and [8], this seems to be the only work concerning a stochastic porous type equation in infinite volume.

We provide a probabilistic representation of solutions to (1.1) extending the results of [6,14] which treated the deterministic case $\mu \equiv 0$. In the deterministic case, it seems that the first author who considered a probabilistic representation (of the type studied in this paper) for the solutions of a non-linear deterministic PDE was McKean [19], particularly in relation with the so called propagation of chaos. In his case, however, the coefficients were smooth. From then on the literature steadily grew and nowadays there is a vast amount of contributions to the subject, see the reference list of $[6,14]$. A probabilistic representation when $\psi(u)=|u| u^{m-1}, m>1$, was provided for instance in [11], in the case of the classical porous media equation. When $m<1$, i.e. in the case of the fast diffusion equation, [9] provides a probabilistic representation of the so called Barenblatt solution, i.e. the solution whose initial condition is concentrated at zero.

$[6,14]$ discussed the probabilistic representation when $\mu=0$ in the so called non-degenerate and degenerate case respectively (see Definition 6.1), where $\psi$ also may have jumps.

In the case $\mu=0$, the equation (1.1) models a non-linear phenomenon macroscopically. Let us denote by $u$ : $[0, T] \times \mathbb{R} \rightarrow \mathbb{R}$ the solution of that equation. The idea of the probabilistic representation is to find a process $\left(Y_{t}, t \in\right.$ $[0, T])$ whose law at time $t$ has $u(t, \cdot)$ for its density. In this case the equation (1.1) is conservative, in the sense that the integral (mass) of the solution is conserved along the time.

The process $Y$ turns out to be the weak solution of the non-linear stochastic differential equation

$$
\left\{\begin{array}{l}
Y_{t}=Y_{0}+\int_{0}^{t} \Phi\left(u\left(s, Y_{s}\right)\right) d B_{s}, \\
\operatorname{Law}\left(Y_{t}\right)=u(t, \cdot), \quad t \geq 0,
\end{array}\right.
$$

where $B$ is a classical Brownian motion. The behavior of $Y$ is the microscopic counterpart of the phenomenon described by (1.1), describing the evolution of a single particle, whose law behaves according to (1.1). 
The idea of this paper is to consider the case when $\mu \neq 0$. This includes the case when $\mu$ is not vanishing but it is deterministic; it happens when only $e^{0}$ is non-zero, and $e^{i} \equiv 0,1 \leq i \leq n$. In this case our technique gives a sort of forward Feynman-Kac formula for a non-linear PDE. One of the main interests of this paper is that it provides a (forward) probabilistic representation for non conservative (random) PDE.

We introduce a doubly stochastic representation on which one can represent the solution of (1.1) as the weightedlaw with respect to the random field $\mu$ (or simply the $\mu$-weighted law) of a solution to a non-linear SDE.

Intuitively, it describes the microscopic aspect of the SPDE (1.1) for almost all quenched $\omega$. The terminology strongly refers to the case where the probability space $(\Omega, \mathcal{F}, P)$ on which the SPDE is defined, remains fixed.

We represent a solution $X$ to (1.1) making use of another independent source of randomness described by another probability space based on some set $\Omega_{1}$.

The analog of the process $Y$, obtained when $\mu$ is zero in [6,14], is a doubly stochastic process, still denoted by $Y$ defined on $\left(\Omega_{1} \times \Omega, Q\right)$, for which, $X$ constitutes the so-called family of $\mu$-marginal weighted laws of $Y$, see Definition 2.4. $Y$ is the solution of a doubly stochastic non-linear diffusion problem, see Definition 3.1. It will be a (doubly) stochastic process $\left(\omega_{1}, \omega\right) \mapsto Y\left(\omega_{1}, \omega\right)$ solution of

$$
Y_{t}=Y_{0}+\int_{0}^{t} \Phi\left(X\left(s, Y_{s}, \omega\right)\right) d B_{s},
$$

and $B(\cdot, \omega)$ is a Brownian motion on $\Omega_{1}$ for almost any fixed $\omega \in \Omega$. The solution of (1.3) is in the following sense: fixing a realization $\omega \in \Omega, Y(\cdot, \omega)$ is a weak solution to the first line of $(1.2)$ with $u(t, \xi)=X(t, \xi, \omega)$. Moreover $X(t, \xi, \omega)$ is the $\mu$-marginal weighted law of $Y_{t}(\cdot, \omega)$.

The paper includes the following main achievements.

1. If we replace in (1.3) $a(s, \xi, \omega)=\Phi(X(s, \xi, \omega))$ and $a$ is bounded and non-degenerate, we show existence and uniqueness of the solution, strongly in $\omega$, weakly in $\omega_{1} \in \Omega_{1}$, see Proposition 4.1. We also show the existence of law densities, for $P$-almost all quenched $\omega$, see Proposition 4.4.

2. Theorem 3.3 states that the $\mu$-marginal weighted laws $X$ of a solution $Y$ of a doubly stochastic non-linear diffusion problem constitute a solution of the stochastic porous media equation (1.1).

3. Conversely, given a solution $X$ of (1.1), under suitable conditions, there is a solution $Y$ of the doubly stochastic non-linear diffusion. This is discussed in Theorem 6.3 and in Theorem 7.1, distinguishing respectively the cases when $\psi$ is non-degenerate and degenerate, see Definition 6.1.

4. When $\psi$ is non-degenerate, then the doubly stochastic non-linear diffusion problem also admits uniqueness, see Theorem 6.3.

5. Section 3.2 illustrates a filtering interpretation for a solution of SPDE (1.1). Indeed, the $\mu$-marginal weighted laws $X$ of a solution $Y$ of a doubly stochastic non-linear diffusion problem (1.3) can be seen as conditional densities of $Y_{t}, t \in[0, T]$ with respect to some probability measure.

6. Uniqueness of the stochastic Fokker-Planck equation obtained replacing $\Phi^{2}$ by a function $a(t, \omega, \xi)$ in $(1.1)$, see Theorem 5.1.

7. Existence of a density to the solution of (1.3), see Proposition 4.4.

\section{Preliminaries}

\subsection{Basic notations}

First we introduce some basic recurrent notations. $\mathcal{M}(\mathbb{R})$ denotes the space of finite real measures.

We recall that $\mathcal{S}(\mathbb{R})$ is the space of the Schwartz fast decreasing test functions. $\mathcal{S}^{\prime}(\mathbb{R})$ is its dual, i.e. the space of Schwartz tempered distributions. On $\mathcal{S}^{\prime}(\mathbb{R})$, the map $(I-\Delta)^{\frac{s}{2}}, s \in \mathbb{R}$, is well-defined. For $s \in \mathbb{R}, H^{s}(\mathbb{R})$ denotes the classical Sobolev space consisting of all functions $f \in \mathcal{S}^{\prime}(\mathbb{R})$ such that $(I-\Delta)^{\frac{s}{2}} f \in L^{2}(\mathbb{R})$. We introduce the norm

$$
\|f\|_{H^{s}}:=\left\|(I-\Delta)^{\frac{s}{2}} f\right\|_{L^{2}},
$$

where $\|\cdot\|_{L^{p}}$ is the classical $L^{p}(\mathbb{R})$-norm for $1 \leq p \leq \infty$. In the sequel, we will often simply denote $H^{-1}(\mathbb{R})$, by $H^{-1}$ and $L^{2}(\mathbb{R})$ by $L^{2}$. Furthermore, $W^{r, p}$ denote the classical Sobolev space of order $r \in \mathbb{N}$ in $L^{p}(\mathbb{R})$ for $1 \leq p \leq \infty$. 
Definition 2.1. Given a function $e$ belonging to $L_{\text {loc }}^{1}(\mathbb{R}) \cap \mathcal{S}^{\prime}(\mathbb{R})$, we say that it is an $H^{-1}$-multiplier, if the map $\varphi \mapsto \varphi e$ is continuous from $\mathcal{S}(\mathbb{R})$ to $H^{-1}$ with respect to the $H^{-1}$-topology on both spaces.

In the following lines we give some other sufficient conditions on a function $e$ to be an $H^{-1}$-multiplier.

Lemma 2.2. Let $e: \mathbb{R} \rightarrow \mathbb{R}$. If $e \in W^{1, \infty}$ (for instance if $\left.e \in W^{2,1}\right)$, then $e$ is a $H^{-1}(\mathbb{R})$-multiplier. In particular the functions $e^{i}, 0 \leq i \leq N$ of Definition 1.1 are $H^{-1}(\mathbb{R})$-multipliers.

Proof. By duality arguments, we observe that it is enough to show the existence of a constant $\mathcal{C}(e)$ such that

$$
\|e g\|_{H^{1}} \leq \mathcal{C}(e)\|g\|_{H^{1}}, \quad \forall g \in \mathcal{S}(\mathbb{R}) .
$$

(2.1) follows by product derivation rules, with for instance $\mathcal{C}(e)=\sqrt{2}\left(\|e\|_{\infty}^{2}+\left\|e^{\prime}\right\|_{\infty}^{2}\right)^{\frac{1}{2}}$.

With respect to the random field $\mu$, we introduce a notation for the Itô type stochastic integral below.

Let $Z=(Z(s, \xi), s \in[0, T], \xi \in \mathbb{R})$ be a random field on $\left(\Omega, \mathcal{F},\left(\mathcal{F}_{t}\right), P\right)$ such that $\int_{0}^{T}\left(\int_{\mathbb{R}}|Z(s, \xi)| \mathrm{d} \xi\right)^{2} \mathrm{~d} s<\infty$ a.s. and it is an $L^{1}(\mathbb{R})$-valued $\left(\mathcal{F}_{S}\right)$-progressively measurable process. Then, the stochastic integral

$$
\int_{[0, t] \times \mathbb{R}} Z(s, \xi) \mu(\mathrm{d} s, \xi) d \xi:=\sum_{i=0}^{N} \int_{0}^{t}\left(\int_{\mathbb{R}} Z(s, \xi) e^{i}(\xi) \mathrm{d} \xi\right) \mathrm{d} W_{s}^{i},
$$

is well-defined.

More generally, if $s \mapsto Z(s, \cdot)$ is a measurable map $[0, T] \times \Omega \mapsto \mathcal{M}(\mathbb{R})$, where $\mathcal{M}(\mathbb{R})$ is the space of signed finite measures, such that $\int_{0}^{T}\|Z(s, \cdot)\|_{\text {var }}^{2} \mathrm{~d} s<\infty$, then the stochastic integral

$$
\int_{[0, t] \times \mathbb{R}} Z(s, \xi) \mu(\mathrm{d} s, \xi) d \xi:=\sum_{i=0}^{N} \int_{0}^{t}\left(\int_{\mathbb{R}} Z(s, \mathrm{~d} \xi)\right) e^{i}(\xi) \mathrm{d} W_{s}^{i},
$$

is well-defined.

We specify now better the filtration $\left(\mathcal{F}_{t}\right)_{t \in[0, T]}$ of the Introduction. We will consider a fixed filtered probability space $\left(\Omega, \mathcal{F}, P,\left(\mathcal{F}_{t}\right)_{t \in[0, T]}\right)$, where $\left(\mathcal{F}_{t}\right)_{t \in[0, T]}$ is the canonical filtration of a standard Brownian motion $\left(W^{1}, \ldots, W^{N}\right)$ enlarged with the $\sigma$-field generated by $x_{0}$. We also suppose that $\mathcal{F}_{0}$ contains the $P$-null sets and $\mathcal{F}=\mathcal{F}_{T}$.

Let $\left(\Omega_{1}, \mathcal{H}\right)$ be a measurable space. In the sequel, we will also consider another filtered probability space $\left(\Omega_{0}, \mathcal{G}, \mathbf{Q},\left(\mathcal{G}_{t}\right)_{t \in[0, T]}\right)$, where $\Omega_{0}=\Omega_{1} \times \Omega, \mathcal{G}=\mathcal{H} \otimes \mathcal{F}$.

Clearly any random element $Z$ on $(\Omega, \mathcal{F})$ will be implicitly extended to $\left(\Omega_{0}, \mathcal{G}\right)$ setting $Z\left(\omega_{1}, \omega\right)=Z(\omega)$. In particular $W^{i}, i=1, \ldots, N$ will be extended in that way.

Here we fix some conventions concerning measurability. Any topological space $E$ is naturally equipped with its Borel $\sigma$-algebra $\mathcal{B}(E)$. For instance $\mathcal{B}(\mathbb{R})(\operatorname{resp} . \mathcal{B}([0, T]))$ denotes the Borel $\sigma$-algebra of $\mathbb{R}$ (resp. $[0, T]$ ).

Given any probability space $(\tilde{\Omega}, \tilde{\mathcal{F}}, \tilde{P})$, the $\sigma$-field $\mathcal{F}$ will always be omitted. When we will say that a map $T$ : $\Omega \times E \rightarrow \mathbb{R}$ is measurable, we will implicitly suppose that the corresponding $\sigma$-algebras are $\mathcal{F} \otimes \mathcal{B}(E)$ and $\mathcal{B}(\mathbb{R})$.

All the processes on any generic measurable space $\left(\Omega_{2}, \mathcal{F}_{2}\right)$ will be considered to be measurable with respect to both variables $(t, \omega)$. In particular any processes on $\Omega_{1} \times \Omega$ is supposed to be measurable with respect to $([0, T] \times$ $\left.\Omega_{1} \times \Omega, \mathcal{B}([0, T]) \otimes \mathcal{H} \otimes \mathcal{F}\right)$.

A function $(A, \omega) \mapsto Q(A, \omega)$ from $\mathcal{H} \times \Omega \rightarrow \mathbb{R}_{+}$is called random kernel (resp. random probability kernel) if for each $\omega \in \Omega, Q(\cdot, \omega)$ is a finite positive (resp. probability) measure and for each $A \in \mathcal{H}, \omega \mapsto Q(A, \omega)$ is $\mathcal{F}$-measurable. The finite measure $Q(\cdot, \omega)$ will also be denoted by $Q^{\omega}$. To that random kernel we can associate a specific finite measure (resp. probability) denoted by $\mathbf{Q}$ on $\left(\Omega_{0}, \mathcal{G}\right)$ setting $\mathbf{Q}(A \times F)=\int_{F} Q(A, \omega) P(\mathrm{~d} \omega)=$ $\int_{F} Q^{\omega}(A) P(\mathrm{~d} \omega)$, for $A \in \mathcal{H}, F \in \mathcal{F}$. The probability $Q$ from above will be supposed here and below to be associated with a random probability kernel. 
Definition 2.3. If there is a measurable space $\left(\Omega_{1}, \mathcal{H}\right)$ and a random kernel $Q$ as before, then the probability space $\left(\Omega_{0}, \mathcal{G}, \mathbf{Q}\right)$ will be called suitable enlarged probability space (of $(\Omega, \mathcal{F}, P)$ ).

As said above, any random variable on $(\Omega, \mathcal{F})$ will be considered as a random variable on $\Omega_{0}=\Omega_{1} \times \Omega$. Then, obviously, $W^{1}, \ldots, W^{N}$ are independent Brownian motions also $\left(\Omega_{0}, \mathcal{G}, Q\right)$.

Given a local martingale $M$ on any filtered probability space, the process $Z:=\mathcal{E}(M)$ denotes its Doléans exponential, which is a local martingale. In particular it is the unique solution of $d Z_{t}=Z_{t-} d M_{t}, Z_{0}=1$. When $M$ is continuous we have $Z_{t}=e^{M_{t}-\frac{1}{2}\langle M\rangle_{t}}$.

\subsection{The concept of marginal weighted laws}

Let us consider a suitably enlarged probability space as in Definition 2.3 .

Definition 2.4. Let $Y: \Omega_{1} \times \Omega \times[0, T] \rightarrow \mathbb{R}$ be a measurable process, progressively measurable on $\left(\Omega_{0}, \mathcal{G}, \mathbf{Q},\left(\mathcal{G}_{t}\right)\right)$, where $\left(\mathcal{G}_{t}\right)$ is some filtration on $\left(\Omega_{0}, \mathcal{G}, \mathbf{Q}\right)$ such that $W^{1}, \ldots, W^{N}$ are $\left(\mathcal{G}_{t}\right)$-Brownian motions on $\left(\Omega_{0}, \mathcal{G}, \mathbf{Q}\right)$. We will make use of the stochastic integral notation

$$
\int_{0}^{t} \mu\left(d s, Y_{s}\right)=\sum_{i=0}^{N} \int_{0}^{t} e^{i}\left(Y_{s}\right) d W_{s}^{i}, \quad t \in[0, T] .
$$

As we shall see below in Proposition 2.6, for every $t \in[0, T]$

$$
E^{\mathbf{Q}}\left(\mathcal{E}_{t}\left(\int_{0}^{\cdot} \mu\left(\mathrm{d} s, Y_{s}\right)\right)\right)<\infty .
$$

To $Y$, we will associate its family of $\mu$-marginal weighted laws (or simply family of $\mu$-weighted laws), i.e. the family of random kernels $(t \in[0, T])$,

$$
\Gamma_{t}=\left(\Gamma_{t}^{Y}(A, \omega), A \in \mathcal{B}(\mathbb{R}), \omega \in \Omega\right)
$$

defined by

$$
\varphi \mapsto E^{Q^{\omega}}\left(\varphi\left(Y_{t}(\cdot, \omega)\right) \mathcal{E}_{t}\left(\int_{0}^{\cdot} \mu\left(\mathrm{d} s, Y_{s}\right)(\cdot, \omega)\right)\right)=\int_{\mathbb{R}} \varphi(r) \Gamma_{t}^{Y}(\mathrm{~d} r, \omega),
$$

where $\varphi$ is a generic bounded real Borel function. We will also say that for fixed $t \in[0, T], \Gamma_{t}$ is the $\mu$-marginal weighted law of $Y_{t}$.

\section{Remark 2.5.}

(i) If $\Omega$ is a singleton $\left\{\omega_{0}\right\}, e^{i}=0,1 \leq i \leq N$, the $\mu$-marginal weighted laws coincide with the weighted laws

$$
\varphi \mapsto E^{\mathbf{Q}}\left(\varphi\left(Y_{t}\right) \exp \left(\int_{0}^{t} e^{0}\left(Y_{s}\right) \mathrm{d} s\right)\right),
$$

with $\mathbf{Q}=Q^{\omega_{0}}$. In particular if $\mu \equiv 0$ then the $\mu$-marginal weighted laws are the classical laws.

(ii) By (2.3), for any $t \in[0, T]$, for $P$ almost all $\omega \in \Omega$,

$$
E^{Q^{\omega}}\left(\mathcal{E}_{t}\left(\int_{0} \mu\left(\mathrm{d} s, Y_{s}\right)(\cdot, \omega)\right)\right)<\infty .
$$

(iii) The function $(t, \omega) \mapsto \Gamma_{t}(A, \omega)$ is measurable, for any $A \in \mathcal{B}(\mathbb{R})$, because $Y$ is a measurable process.

(iv) In the case $e^{0}=0$, the situation is the following. For each fixed $\omega \in \Omega,(2.4)$ is a (random) non-negative measure which is not a probability. However the expectation of its total mass is indeed 1 . 
Proposition 2.6. Consider the situation of Definition 2.4. Then we have the following.

(i) The process $M_{t}:=\mathcal{E}_{t}\left(\sum_{i=1}^{N} \int_{0}^{e} e^{i}\left(Y_{s}\right) \mathrm{d} W_{s}^{i}\right)$ is a martingale. We emphasize that the sum starts indeed at $i=1$.

(ii) The quantity (2.3) is bounded by $\exp \left(T\left\|e^{0}\right\|_{\infty}\right)$.

(iii) $E^{\mathbf{Q}_{(}}\left(M_{t}^{2}\right) \leq \exp \left(3 T \sum_{i=1}^{N}\left\|e^{i}\right\|_{\infty}^{2}\right), t \in[0, T]$. Consequently $M$ is a uniformly integrable martingale.

(iv) For $P$-a.e. $\omega \in \Omega$, $\sup _{0 \leq t \leq T}\left\|\Gamma_{t}(\cdot, \omega)\right\|_{\mathrm{var}}<\infty$, where we remind that $\|\cdot\|_{\mathrm{var}}$ stands for the total variation.

Remark 2.7. Proposition 2.6(ii) yields in particular that $Y$ always admits $\mu$-marginal weighted laws.

\section{Proof of Proposition 2.6.}

(i) The result follows since the Novikov condition $E^{\mathbf{Q}}\left(\exp \left(\frac{1}{2} \sum_{i=1}^{N} \int_{s}^{t} e^{i}\left(Y_{s}\right)^{2} \mathrm{~d} s\right)\right)<\infty$ is verified, because the functions $e^{i}, i=1, \ldots, N$, are bounded.

(ii) This follows because $E^{\mathbf{Q}}\left(M_{t}\right)=1, \forall t \in[0, T]$.

(iii) $M_{t}^{2}$ is equal to $N_{t} \exp \left(3 \sum_{i=1}^{N} \int_{0}^{t}\left(e^{i}\right)^{2}\left(Y_{s}\right) \mathrm{d} s\right)$, where $N$ is a positive martingale with $N_{0}=1$.

(iv) For $t \in[0, T]$,

$$
\begin{aligned}
\sup _{t \leq T}\left\|\Gamma_{t}(\cdot, \omega)\right\|_{\mathrm{var}} & =\sup _{t \leq T} E^{Q^{\omega}}\left(M_{t} \exp \left(\int_{0}^{t} e^{0}\left(Y_{s}\right) \mathrm{d} s\right)\right) \\
& \leq \exp \left(T\left\|e^{0}\right\|_{\infty}\right) \sup _{t \leq T} E^{Q^{\omega}}\left(M_{t}\right) .
\end{aligned}
$$

Taking the expectation with respect to $P$ it implies

$$
\begin{aligned}
E^{P}\left(\sup _{t \leq T}\left\|\Gamma_{t}^{Y}(\cdot, \omega)\right\|_{\mathrm{var}}\right) & \leq \exp \left(T\left\|e^{0}\right\|_{\infty}\right) E^{P}\left(\sup _{t \leq T} E^{Q^{\omega}}\left(M_{t}\right)\right) \\
& \leq \exp \left(T\left\|e^{0}\right\|_{\infty}\right) E^{P}\left(E^{Q^{\omega}}\left(\sup _{t \leq T} M_{t}\right)\right) .
\end{aligned}
$$

By the Burkholder-Davis-Gundy (BDG) inequality this is bounded by

$$
\begin{aligned}
3 \exp \left(T\left\|e^{0}\right\|_{\infty}\right) E^{\mathbf{Q}}\left(\langle M\rangle_{T}^{\frac{1}{2}}\right) & \leq 3 \exp \left(T\left\|e^{0}\right\|_{\infty}\right) E^{\mathbf{Q}}\left(\left[\int_{0}^{T} \mathrm{~d} s \sum_{i=1}^{N} M_{s}^{2} e^{i}\left(Y_{S}\right)^{2}\right]^{\frac{1}{2}}\right) \\
& \leq \mathrm{C}(e, N, T)\left\{E^{\mathbf{Q}}\left(\int_{0}^{T} \mathrm{~d} s M_{s}^{2}\right)\right\}^{\frac{1}{2}},
\end{aligned}
$$

where the last inequality is due to Jensen's inequality; $\mathrm{C}(e, N, T)$ is a constant depending on $N, T$ and $e^{i}, i=$ $0, \ldots, N$. By Fubini's theorem and item (iii), we have

$$
E^{\mathbf{Q}}\left(\int_{0}^{T} \mathrm{~d} s M_{s}^{2}\right) \leq T \exp \left(3 T \sum_{i=1}^{N}\left\|e^{i}\right\|_{\infty}\right) .
$$

The lemma below gives a characterization of the $\mu$-weighted laws of a process $Y$ living on an enlarged probability space.

Lemma 2.8. Let $Y$ (resp. $\tilde{Y})$ be a process on a suitable enlarged probability space $\left(\Omega_{0}, \mathcal{G}, \mathbf{Q}\right)\left(\right.$ resp. $\left.\left(\tilde{\Omega}_{0}, \tilde{\mathcal{G}}, \tilde{\mathbf{Q}}\right)\right)$. Set $W=\left(W^{1}, \ldots, W^{N}\right)$. Suppose that the law of $(Y, W)$ under $\mathbf{Q}$ and the law of $(\tilde{Y}, W)$ under $\tilde{\mathbf{Q}}$ are the same. Then, the $\mu$-marginal weighted laws of $Y$ under $\mathbf{Q}$ coincide a.s. with the $\mu$-marginal weighted laws of $\tilde{Y}$ under $\tilde{\mathbf{Q}}$. 
Proof. Let $0 \leq t \leq T$. Using the assumption, we deduce that for any bounded continuous function $f: \mathbb{R} \rightarrow \mathbb{R}$, and every $F \in \mathcal{F}_{t}$, we have

$$
E^{\mathbf{Q}}\left(1_{F} f\left(Y_{t}\right) \mathcal{E}_{t}\left(\sum_{i=0}^{N} \int_{0}^{\cdot} e^{i}\left(Y_{s}\right) d W_{s}^{i}\right)\right)=E^{\tilde{\mathbf{Q}}}\left(1_{F} f\left(\tilde{Y}_{t}\right) \mathcal{E}_{t}\left(\sum_{i=0}^{N} \int_{0}^{\cdot} e^{i}\left(\tilde{Y}_{s}\right) d W_{s}^{i}\right)\right) .
$$

To show this, using classical regularization properties of Itô integral, see e.g. Theorem 2 in [25], and uniform integrability arguments, we first observe that

$$
\mathcal{E}_{t}\left(\sum_{i=0}^{N} \int_{0}^{\cdot} e^{i}\left(Y_{s}\right) d W_{s}^{i}\right)
$$

is the limit in $L^{2}\left(\Omega_{0}, \mathbf{Q}\right)$ of

$$
\mathcal{E}_{t}\left(\sum_{i=0}^{N} \int_{0}^{.} e^{i}\left(Y_{s}\right) \frac{W_{s+\varepsilon}^{i}-W_{s}^{i}}{\varepsilon} \mathrm{d} s\right)
$$

A similar approximation property arises replacing $Y$ with $\tilde{Y}$ and $\mathbf{Q}$ with $\tilde{\mathbf{Q}}$. Then (2.5) easily follows.

To conclude, it will be enough to show the existence of a countable family $\left(f_{j}\right)_{j \in \mathbb{N}}$ of bounded continuous real functions for which, for $P$ almost all $\omega \in \Omega$, for any $j \in \mathbb{N}$, we have $R_{j}=\tilde{R}_{j}$ where

$$
\begin{aligned}
& R_{j}(\omega)=E^{Q^{\omega}}\left(f_{j}\left(Y_{t}(\cdot, \omega)\right) \mathcal{E}_{t}\left(\sum_{i=0}^{N} \int_{0}^{\cdot} e^{i}\left(Y_{s}(\cdot, \omega)\right) d W_{s}^{i}\right)\right), \\
& \tilde{R}_{j}(\omega)=E^{\tilde{Q}^{\omega}}\left(f_{j}\left(\tilde{Y}_{t}(\cdot, \omega)\right) \mathcal{E}_{t}\left(\sum_{i=0}^{N} \int_{0}^{\cdot} e^{i}\left(\tilde{Y}_{s}(\cdot, \omega)\right) d W_{s}^{i}\right)\right) .
\end{aligned}
$$

This will follow, since applying (2.5), for any $F \in \mathcal{F}_{t}$, we have $E^{P}\left(1_{F} R_{j}\right)=E^{P}\left(1_{F} \tilde{R}_{j}\right)$.

\subsection{SPDE, weak-strong existence of SDEs}

In this section we introduce the basic concepts related to the stochastic porous media equation and the related nonlinear diffusion.

Definition 2.9. A random field $X=(X(t, \xi, \omega), t \in[0, T], \xi \in \mathbb{R}, \omega \in \Omega)$ is said to be a solution to (1.1) if $P$ a.s. we have the following.

1. $X \in C\left([0, T] ; \mathcal{S}^{\prime}(\mathbb{R})\right) \cap L^{2}\left([0, T] ; L_{\text {loc }}^{1}(\mathbb{R})\right)$.

2. $X$ is an $\mathcal{S}^{\prime}(\mathbb{R})$-valued $\left(\mathcal{F}_{t}\right)$-progressively measurable process.

3. For any test function $\varphi \in \mathcal{S}(\mathbb{R})$ with compact support, $t \in] 0, T]$ a.s. we have

$$
\begin{aligned}
\int_{\mathbb{R}} X(t, \xi) \varphi(\xi) \mathrm{d} \xi= & \int_{\mathbb{R}} x_{0}(\mathrm{~d} \xi) \varphi(\xi)+\frac{1}{2} \int_{0}^{t} \mathrm{~d} s \int_{\mathbb{R}} \psi(X(s, \xi, \cdot)) \varphi^{\prime \prime}(\xi) \mathrm{d} \xi \\
& +\int_{[0, t] \times \mathbb{R}} X(s, \xi) \varphi(\xi) \mu(\mathrm{d} s, \xi) \mathrm{d} \xi
\end{aligned}
$$

At Definition 3.1, we will present the concept of double stochastic non-linear diffusion which is a McKean type equation with a supplementary source of randomness. Before this, as a first step, we will introduce a particular the case of simple double stochastic differential equation (DSDE). Let $\gamma:[0, T] \times \mathbb{R} \times \Omega \rightarrow \mathbb{R}$ be an $\left(\mathcal{F}_{t}\right)$-progressively measurable random fields and $x_{0}$ be a probability on $\mathcal{B}(\mathbb{R})$. 


\section{Definition 2.10.}

(a) We say that (DSDE) $\left(\gamma, x_{0}\right)$ admits weak-strong existence if there is a suitable extended probability space $\left(\Omega_{0}, \mathcal{G}, \mathbf{Q}\right)$, i.e. a measurable space $\left(\Omega_{1}, \mathcal{H}\right)$, a probability kernel $(Q(\cdot, \omega), \omega \in \Omega)$ on $\mathcal{H} \times \Omega$, two Q-a.s. continuous processes $Y, B$ on $\left(\Omega_{0}, \mathcal{G}\right)$ where $\Omega_{0}=\Omega_{1} \times \Omega, \mathcal{G}=\mathcal{H} \otimes \mathcal{F}$ such that the following holds.

(1) For almost all $\omega, Y(\cdot, \omega)$ is a (weak) solution to

$$
\left\{\begin{array}{l}
Y_{t}(\cdot, \omega)=Y_{0}+\int_{0}^{t} \gamma\left(s, Y_{s}(\cdot, \omega), \omega\right) \mathrm{d} B_{s}(\cdot, \omega), \\
\operatorname{Law}\left(Y_{0}\right)=x_{0},
\end{array}\right.
$$

with respect to $Q^{\omega}$, where $B(\cdot, \omega)$ is a $Q^{\omega}$-Brownian motion for almost all $\omega$.

(2) We denote $\left(\mathcal{Y}_{t}\right)$ the canonical filtration associated with $\left(Y_{s}, 0 \leq s \leq t\right)$ and $\mathcal{G}_{t}=\mathcal{Y}_{t} \vee\left(\left\{\varnothing, \Omega_{1}\right\} \otimes \mathcal{F}_{t}\right)$. $W^{1}, \ldots, W^{N}$ is a $\left(\mathcal{G}_{t}\right)$-martingale under $\mathbf{Q}$.

(3) For every $0 \leq s \leq T$, for every bounded continuous $\mathcal{A}: C([0, s]) \rightarrow \mathbb{R}$, the r.v. $\omega \mapsto E^{Q^{\omega}}\left(\mathcal{A}\left(Y_{r}(\cdot, \omega), r \in\right.\right.$ $[0, s]))$ is $\mathcal{F}_{s}$-measurable.

(b) We say that (DSDE) $\left(\gamma, x_{0}\right)$ admits weak-strong uniqueness if the following holds. Consider a measurable space $\left(\Omega_{1}, \mathcal{H}\right)\left(\operatorname{resp} .\left(\widetilde{\Omega}_{1}, \widetilde{\mathcal{H}}\right)\right)$, a probability kernel $(Q(\cdot, \omega), \omega \in \Omega)(\operatorname{resp} .(\widetilde{Q}(\cdot, \omega), \omega \in \Omega))$, with processes $(Y, B)$ (resp. $(\widetilde{Y}, \widetilde{B})$ ) such that $(2.6)$ holds (resp. $(2.6)$ holds with $\left(\Omega_{0}, \mathcal{G}, \mathbf{Q}\right)$ replaced with $\left(\widetilde{\Omega}_{0}, \widetilde{\mathcal{G}_{0}}, \widetilde{\mathbf{Q}}\right), \widetilde{\mathbf{Q}}$ being associated with $(\widetilde{Q}(\cdot, \omega)))$. Moreover we suppose that item (2) is verified for $Y$ and $\tilde{Y}$.

Then $\left(Y, W^{1}, \ldots, W^{N}\right)$ and $\left(\widetilde{Y}, W^{1}, \ldots, W^{N}\right)$ have the same law.

(c) A process $Y$ fulfilling items (1) and (2) under (a) will be called weak-strong solution of (DSDE) $\left(\gamma, x_{0}\right)$.

Remark 2.11. Let $Y$ be a weak-strong solution of $(\mathrm{DSDE})\left(\gamma, x_{0}\right)$ with corresponding $B$.

(a) Since for almost all $\omega \in \Omega, B(\cdot, \omega)$ is a Brownian motion under $Q^{\omega}$, it is clear that $B$ is a Brownian motion under $Q$, which is independent of $\mathcal{F}_{T}$, i.e. independent of $W^{1}, \ldots, W^{N}$.

Indeed let $\mathcal{A}: C([0, T]) \rightarrow \mathbb{R}$ be a continuous bounded functional, and denote by $\mathcal{W}$ the Wiener measure on $C([0, T])^{N}$. Let $F$ be a bounded $\mathcal{F}_{T}$-measurable r.v. Since for each $\omega, B(\cdot, \omega)$ is a Wiener process with respect to $Q^{\omega}$, we get

$$
\begin{aligned}
E^{\mathbf{Q}}(F \mathcal{A}(B)) & =\int_{\Omega} F E^{Q^{\omega}}(\mathcal{A}(B(\cdot, \omega))) \mathrm{d} P(\omega) \\
& =\int_{\Omega} F(\omega) \mathrm{d} P(\omega) \int_{\Omega_{1}} \mathcal{A}\left(\omega_{1}\right) d \mathcal{W}\left(\omega_{1}\right) \\
& =\int_{\Omega_{0}} F(\omega) \mathrm{d} \mathbf{Q}\left(\omega_{0}\right) \int_{\Omega_{0}} \mathcal{A}\left(\omega_{1}\right) \mathrm{d} \mathbf{Q}\left(\omega_{0}\right) .
\end{aligned}
$$

This shows that $\left(W^{1}, \ldots, W^{N}\right)$ and $B$ are independent. Taking $F=1_{\Omega}$ in previous expression, the equality between the left-hand side and the third term, shows that $B$ is a Brownian motion under $Q$.

(b) Since for any $1 \leq i, j \leq N$,

$$
\left[W^{i}, W^{j}\right]_{t}=\delta_{i j} t, \quad\left[W^{i}, B\right]=0, \quad[B, B]_{t}=t,
$$

Lévy's characterization theorem, implies that $\left(W^{1}, \ldots, W^{N}, B\right)$ is a Q-Brownian motion.

(c) An equivalent formulation to (1) in item (a) of Definition 2.10 is the following. For $P$ a.e., $\omega \in \Omega, Y(\cdot, \omega)$ solves the $Q^{\omega}$-martingale problem with respect to the (random) PDE operator

$$
L_{t}^{\omega} f(\xi)=\frac{1}{2} \gamma^{2}(t, \xi, \omega) f^{\prime \prime}(\xi)
$$

and initial distribution $x_{0}$. Indeed, we remark that the construction can be performed on the canonical space $\Omega_{1}=C([0, T] ; \mathbb{R})$. 
Proposition 2.12. Let $Y$ be a process as in Definition 2.10(a). We have the following.

1. $Y$ is a $\left(\mathcal{G}_{t}\right)$-martingale on the product space $\left(\Omega_{0}, \mathcal{G}, \mathbf{Q}\right)$.

2. $\left[Y, W^{i}\right]=0, \forall 1 \leq i \leq N$.

Proof. Let $0 \leq s<t \leq T, F_{s} \in \mathcal{F}_{s}$ and $G: C([0, s]) \rightarrow \mathbb{R}$ be continuous and bounded. We will prove below that, for $1 \leq i \leq N+1$, setting $W_{t}^{N+1}=1$, for all $t \geq 0$,

$$
E^{\mathbf{Q}}\left(Y_{t} W_{t}^{i} G\left(Y_{r}, r \leq s\right) 1_{F_{s}}\right)=E^{\mathbf{Q}}\left(Y_{s} W_{s}^{i} 1_{F_{s}} G\left(Y_{r}, r \leq s\right)\right) .
$$

Then (2.7) with $i=N+1$ shows item 1 . Considering (2.7) with $1 \leq i \leq N$, shows that $Y W^{i}$ is a $\left(\mathcal{G}_{t}\right)$-martingale, which shows item 2 . Therefore, it remains to show (2.7).

The left-hand side of that equality gives

$$
\begin{aligned}
& \int_{\Omega} \mathrm{d} P(\omega) W_{t}^{i}(\omega) 1_{F_{s}}(\omega) E^{Q^{\omega}}\left(Y_{t}(\cdot, \omega) G\left(Y_{r}(\cdot, \omega), r \leq s\right)\right) \\
& \quad=\int_{\Omega} \mathrm{d} P(\omega) 1_{F_{s}}(\omega) W_{t}^{i}(\omega) E^{Q^{\omega}}\left(Y_{s}(\cdot, \omega) G\left(Y_{r}(\cdot, \omega), r \leq s\right)\right),
\end{aligned}
$$

because $Y(\cdot, \omega)$ is a $Q^{\omega}$-martingale for $P$-almost all $\omega$. To obtain the right-hand side of (2.7) it is enough to remember that $W^{i}$ are $\left(\mathcal{G}_{t}\right)$-martingales and that item (a)(3) in Definition 2.10 holds. This concludes the proof of Proposition 2.12 .

Remark 2.13. Lemma 2.8 shows that, whenever weak-strong uniqueness holds, then the $\mu$-weighted marginal laws of any weak solution $Y$ are uniquely determined.

\section{The concept of doubly probabilistic representation}

\subsection{The doubly stochastic non-linear diffusion}

We come back to the notations and conventions of the Introduction and of Section 2 . Let $x_{0}$ be a probability on $\mathbb{R}$. The doubly probabilistic representation is based on the following idea. Let $Y: \Omega_{0} \times[0, T] \rightarrow \mathbb{R}$ be a measurable process where $\Omega_{0}=\Omega_{1} \times \Omega$ is the usual enlarged probability space as introduced in Definition 2.3. Let $\mathbf{Q}$ be a probability inherited from a random kernel $Q^{\omega}$ as before Definition 2.3. Let $\left(\mathcal{G}_{t}\right)$, where $\left(\mathcal{G}_{t}\right)$ is some filtration on $\left(\Omega_{0}, \mathcal{G}\right)$ such that $W^{1}, \ldots, W^{N}$ are $\left(\mathcal{G}_{t}\right)$-Brownian motions on $\left(\Omega_{0}, \mathcal{G}, \mathbf{Q}\right)$.

Suppose that

$$
\left\{\begin{array}{l}
Y_{t}=Y_{0}+\int_{0}^{t} \Phi\left(X\left(s, Y_{s}\right)\right) d B_{s}, \\
\left.\left.\mu \text {-Weighted Law }\left(Y_{t}\right)=X(t, \xi) \mathrm{d} \xi, \quad t \in\right] 0, T\right], \\
\mu \text {-Weighted Law }\left(Y_{0}\right)=x_{0}(\mathrm{~d} \xi),
\end{array}\right.
$$

where $B$ is a $Q$-standard Brownian motion with respect to $\left(\mathcal{G}_{t}\right)$. Then $X$ solves the SPDE (1.1). This will be the object of Theorem 3.3. Vice versa, if $X$ is a solution of (1.1) then there is a process $Y$ solving (3.1), see Theorem 7.1.

\section{Definition 3.1.}

(1) We say that the doubly stochastic non-linear diffusion (DSNLD) driven by $\Phi$ (on the space $(\Omega, \mathcal{F}, P)$ ) with initial condition $x_{0}$, related to the random field $\mu$ (shortly (DSNLD) $\left(\Phi, \mu, x_{0}\right)$ ) admits weak existence if there is a measurable random field $X:[0, T] \times \mathbb{R} \times \Omega \rightarrow \mathbb{R}$ with the following properties.

(a) The problem (DSDE) $\left(\gamma, x_{0}\right)$ with $\gamma(t, \xi, \omega)=\Phi(X(t, \xi, \omega))$ admits weak-strong existence.

(b) $X=X(t, \xi, \cdot) \mathrm{d} \xi, t \in] 0, T]$, is the family of $\mu$-marginal weighted laws of $Y$, where $Y$ is the solution of (2.6) in Definition 2.10. In other words $X$ constitutes the densities of those $\mu$-marginal weighted laws. 
(2) A couple $(Y, X)$, such that $Y$ is a (weak-strong) solution to the $(\operatorname{DSDE})\left(\gamma, x_{0}\right)$, is called weak solution to the $(\mathrm{DSNLD})\left(\Phi, \mu, x_{0}\right) . Y$ is also called doubly stochastic representation of the random field $X$.

(3) Suppose that, given two measurable random fields $X^{i}:[0, T] \times \mathbb{R} \times \Omega \rightarrow \mathbb{R}, i=1,2$ on $\left(\Omega, \mathcal{F}, P,\left(\mathcal{F}_{t}\right)\right.$ ), and $Y^{i}$, on extended probability space $\left(\Omega_{0}^{i}, \mathbf{Q}^{i}\right), i=1,2$, such that $\left(Y^{i}, X^{i}\right)$ is a weak-strong solution of $(\operatorname{DSDE})\left(\Phi\left(X^{i}\right), x_{0}\right), i=1,2$, where we always have that $\left(Y^{1}, W^{1}, \ldots, W^{N}\right)$ and $\left(Y^{2}, W^{1}, \ldots, W^{N}\right)$ have the same law. Then we say that the $(\mathrm{DSNLD})\left(\Phi, \mu, x_{0}\right)$ admits weak uniqueness.

Remark 3.2. If (DSNLD) $\left(\Phi, \mu, x_{0}\right)$ admits weak uniqueness then the $\mu$-marginal weighted laws of $Y$ are uniquely determined, $P$-a.s., see Lemma 2.8 .

Theorem 3.3. Let $(Y, X)$ be a solution of $(D S N L D)\left(\Phi, \mu, x_{0}\right)$. Then $X$ is a solution to the SPDE (1.1).

\section{Remark 3.4.}

1. Let $t \in[0, T]$. Let $\varphi: \mathbb{R} \rightarrow \mathbb{R}$ be Borel and bounded. Then

$$
\int_{\mathbb{R}} \varphi(\xi) X(t, \xi, \omega) \mathrm{d} \xi=E^{Q^{\omega}}\left(\varphi\left(Y_{t}(\omega)\right) \mathcal{E}_{t}\left(\int_{0}^{\cdot} \mu\left(d s, Y_{s}(\omega)\right)\right)\right) .
$$

So

$$
\int_{\mathbb{R}} X(t, \xi, \omega) d \xi=E^{Q^{\omega}}\left(\mathcal{E}_{t}\left(\int_{0}^{\cdot} \mu\left(d s, Y_{S}(\omega)\right)\right)\right) .
$$

Even though for a.e. $\omega \in \Omega$, the previous expression is not necessarily a probability measure, of course,

$$
v_{\omega}: \varphi \mapsto \frac{\int_{\mathbb{R}} \varphi(\xi) X(t, \xi, \omega) d \xi}{\int_{\mathbb{R}} X(t, \xi, \omega) d \xi}
$$

is one. It can be expressed as

$$
v_{\omega}(A)=\frac{E^{Q^{\omega}}\left(1_{A}\left(Y_{t}\right) \mathcal{E}_{t}(M(\cdot, \omega))\right)}{E^{Q^{\omega}} \mathcal{E}_{t}(M(\cdot, \omega))},
$$

where $M_{t}(\cdot, \omega)=\int_{0}^{t} \mu\left(d s, Y_{s}(\cdot, \omega)\right), t \in[0, T]$, is defined in (2.2).

2. Consider the particular case $e_{0}=0, e_{1}=c, c$ being some constant. In this case, the $\mu$-marginal laws are given by

$$
A \mapsto E^{Q^{\omega}}\left(1_{A}\left(Y_{t}\right) c \mathcal{E}_{t}(W)\right)=c \mathcal{E}_{t}(W) E^{Q^{\omega}}\left(1_{A}\left(Y_{t}\right)\right)=c \mathcal{E}_{t}(W) \nu_{\omega}(t, A)
$$

and $v_{\omega}(t, \cdot)$ is the law of $Y_{t}(\cdot, \omega)$ under $Q^{\omega}$.

Proof of Theorem 3.3. Let $B$ denote the Brownian motion associated to $Y$ as a solution to (DSDE) $\left(\gamma, x_{0}\right)$, mentioned in item (a)(1) of Definition 3.1. For $t \in[0, T]$, we set

$$
Z_{t}=\mathcal{E}_{t}\left(\int_{0}^{\cdot} \mu\left(\mathrm{d} s, Y_{s}\right)\right), \quad M_{t}=Z_{t} \exp \left(-\int_{0}^{t} e^{0}\left(Y_{s}\right) \mathrm{d} s\right), \quad t \in[0, T]
$$

1. Proof of Definition 2.9 1. By Proposition 2.6, $\left(M_{t}, t \in[0, T]\right)$ is a uniformly integrable martingale. Consequently $t \mapsto Z_{t}$ is continuous in $L^{1}\left(\Omega_{0}, \mathbf{Q}\right)$. On the other hand the process $Y$ is continuous. This implies that $P$ a.e. $\omega \in \Omega$, $X \in C([0, T] ; \mathcal{M}(\mathbb{R}))$, where $\mathcal{M}(\mathbb{R})$ is equipped with the weak topology. This implies that $X \in C\left([0, T] ; \mathcal{S}^{\prime}(\mathbb{R})\right)$. Furthermore, for $P$ a.e. $\omega \in \Omega$, and $t \in] 0, T], X(t, \cdot, \omega) \in L^{1}(\mathbb{R})$ and $\int_{\mathbb{R}} X(t, \xi, \omega) \mathrm{d} \xi=\|\Gamma(t, \cdot, \omega)\|_{\text {var. }}$. By Proposition 2.6(iv), it follows that $P$-a.s. $X(\cdot, \cdot, \omega) \in L^{\infty}\left([0, T] ; L^{1}(\mathbb{R})\right) \subset L^{2}\left([0, T] ; L_{\text {loc }}^{1}(\mathbb{R})\right)$.

2. Definition 2.9 2. follows from Remark 3.4 1. and Definition 2.10(a)(3). 
3. Proof of Definition 2.93. Let $\varphi \in \mathcal{S}(\mathbb{R})$ with compact support. Taking into account Proposition 2.12, we apply Itô's formula to get

$$
\begin{aligned}
\varphi\left(Y_{t}\right) Z_{t}= & \varphi\left(Y_{0}\right)+\int_{0}^{t} \varphi^{\prime}\left(Y_{s}\right) Z_{s} \mathrm{~d} Y_{s}+\int_{0}^{t} \varphi\left(Y_{s}\right) Z_{s}\left(\mu\left(\mathrm{d} s, Y_{s}\right)-\frac{1}{2} \sum_{i=1}^{N}\left(e^{i}\left(Y_{s}\right)\right)^{2} \mathrm{~d} s\right) \\
& +\frac{1}{2} \int_{0}^{t} \varphi^{\prime \prime}\left(Y_{s}\right) \Phi^{2}\left(X\left(s, Y_{s}\right)\right) Z_{s} \mathrm{~d} s+\frac{1}{2} \int_{0}^{t} \varphi\left(Y_{s}\right) Z_{s}\left(\sum_{i=1}^{N}\left(e^{i}\left(Y_{s}\right)\right)^{2}\right) \mathrm{d} s .
\end{aligned}
$$

Indeed we remark that $\int_{0}^{t} \varphi^{\prime}\left(Y_{s}\right) \mathrm{d}[Z, Y]_{s}=0$, because $[Z, Y]_{t}=\sum_{i=1}^{N} \int_{0}^{t} e^{i}\left(Y_{s}\right) Z_{s} \mathrm{~d}\left[W^{i}, Y\right]_{s}=0$; in fact $\left[W^{i}, Y\right]=0$ by Proposition 2.12. So

$$
\begin{aligned}
\varphi\left(Y_{t}\right) Z_{t}= & \varphi\left(Y_{0}\right)+\int_{0}^{t} \varphi^{\prime}\left(Y_{s}\right) Z_{s} \Phi\left(X\left(s, Y_{s}\right)\right) \mathrm{d} B_{s} \\
& +\int_{0}^{t} \varphi\left(Y_{s}\right) Z_{s} \mu\left(\mathrm{d} s, Y_{s}\right)+\frac{1}{2} \int_{0}^{t} \varphi^{\prime \prime}\left(Y_{s}\right) \Phi^{2}\left(X\left(s, Y_{s}\right)\right) Z_{s} \mathrm{~d} s .
\end{aligned}
$$

Taking the expectation with respect to $Q^{\omega}$ we get $\mathrm{d} P$-a.s.,

$$
\begin{aligned}
\int_{\mathbb{R}} \mathrm{d} \xi \varphi(\xi) X(t, \xi)= & \int_{\mathbb{R}} \varphi(\xi) x_{0}(\mathrm{~d} \xi)+\sum_{i=0}^{N} \int_{0}^{t} \mathrm{~d} W_{s}^{i}\left(\int_{\mathbb{R}} \mathrm{d} \xi \varphi(\xi) e^{i}(\xi) X(s, \xi)\right) \\
& +\frac{1}{2} \int_{0}^{t} \mathrm{~d} s \int_{\mathbb{R}} \mathrm{d} \xi \varphi^{\prime \prime}(\xi) \Phi^{2}(X(s, \xi)) X(s, \xi),
\end{aligned}
$$

which implies the result. Indeed, in the previous equality, we have used Lemma 3.5 below.

Lemma 3.5. Let $1 \leq i \leq N$. For $P$ a.e. $\omega \in \Omega$, we have

$$
E^{Q^{\omega}}\left(\int_{0}^{t} \varphi\left(Y_{s}\right) Z_{s} e^{i}\left(Y_{s}\right) \mathrm{d} W_{s}^{i}\right)(\cdot, \omega)=\int_{0}^{t} \mathrm{~d} W_{s}^{i}(\omega) \int_{\mathbb{R}} \varphi(\xi) e^{i}(\xi) X(s, \xi, \omega) \mathrm{d} \xi
$$

Proof. Since the Brownian motions $W^{i}$ are not random for $Q^{\omega}$, it is possible to justify the permutation of the stochastic integral with respect to $W^{i}$ and $E^{Q^{\omega}}$ by a Fubini argument approximating the stochastic integrals via Lebesgue integral, see e.g. Theorem 2 of [25]. A complete proof is given in [7].

\subsection{Filtering interpretation}

Item 1. of Remark 3.4 has an interpretation in the framework of filtering theory, see e.g. [20] for a comprehensive introduction on that subject.

Suppose $e^{0}=0$. Let $\hat{\mathbf{Q}}$ be a probability on some probability space $\left(\Omega_{0}, \mathcal{G}_{T}\right)$, and consider the non-linear diffusion problem (1.2) as a basic dynamical phenomenon. We suppose now that there are $N$ observations $Y^{1}, \ldots, Y^{N}$ related to the process $Y$ generating a filtration $\left(\mathcal{F}_{t}\right)$. We suppose in particular that $\mathrm{d} Y_{t}^{i}=\mathrm{d} W_{t}^{i}+e^{i}\left(Y_{t}\right) \mathrm{d} t, 1 \leq i \leq N$, and $W^{1}, \ldots, W^{N}$ be $\left(\mathcal{F}_{t}\right)$-Brownian motions. Consider the following dynamical system of non-linear diffusion type:

$$
\left\{\begin{array}{l}
Y_{t}=Y_{0}+\int_{0}^{t} \Phi\left(X\left(s, Y_{s}\right)\right) d B_{s}, \\
\mathrm{~d} Y_{t}^{i}=\mathrm{d} W_{t}^{i}+e^{i}\left(Y_{t}\right) \mathrm{d} t, \quad 1 \leq i \leq N, \\
X(t, \cdot): \text { conditional law of } Y_{t} \text { under } \mathcal{F}_{t} .
\end{array}\right.
$$


The third equality of (3.2) means, under $\mathbf{Q}$, that we have,

$$
\int_{\mathbb{R}} \varphi(\xi) X(t, \xi) \mathrm{d} \xi=E\left(\varphi\left(Y_{t}\right) \mid \mathcal{F}_{t}\right) .
$$

We remark that, under the new probability $Q$ defined by $\mathrm{d} \mathbf{Q}=\mathrm{d} \hat{\mathbf{Q}} \mathcal{E}\left(\int_{0}^{T} \mu\left(\mathrm{d} s, Y_{s}\right)\right), Y^{1}, \ldots, Y^{N}$ are standard $\left(\mathcal{F}_{t}\right)$ independent Brownian motions. Then (3.3) becomes

$$
\int_{\mathbb{R}} \varphi(\xi) X(t, \xi) \mathrm{d} \xi=E^{\hat{\mathbf{Q}}}\left(\varphi\left(Y_{t}\right) \mid \mathcal{F}_{t}\right)=\frac{E^{\mathbf{Q}}\left(\varphi\left(Y_{t}\right) \mathcal{E}_{t}\left(\int_{0}^{\cdot} \mu\left(d s, Y_{s}\right) \mid \mathcal{F}_{t}\right)\right)}{E^{\mathbf{Q}}\left(\mathcal{E}_{t}\left(\int_{0}^{\cdot} \mu\left(d s, Y_{s}\right) \mid \mathcal{F}_{t}\right)\right)} .
$$

Consequently, by Theorem 3.3, $X$ will be the solution of the SPDE (1.1), with $x_{0}$ being the law of $Y_{0}$; so (1.1) constitutes the Zakai type equation associated with our filtering problem.

\section{The densities of the $\mu$-marginal weighted laws}

This section constitutes an important step towards the doubly probabilistic representation of a solution to (1.1), when $\psi$ is non-degenerate. Let $x_{0}$ be a fixed probability on $\mathbb{R}$. We recall that a process $Y$ (on a suitable enlarged probability space $\left.\left(\Omega_{0}, \mathcal{G}, \mathbf{Q}\right)\right)$, which is a weak solution to the $\operatorname{DSNLD}\left(\Phi, \mu, x_{0}\right)$, is in particular a weak-strong solution of a $(\mathrm{DSDE})\left(\gamma, x_{0}\right)$ where $\gamma:[0, T] \times \mathbb{R} \times \Omega \rightarrow \mathbb{R}$ is some suitable progressively measurable random field on $(\Omega, \mathcal{F}, P)$. The aim of this section is twofold.

(A) To show that whenever $\gamma$ is a.s. bounded and non-degenerate, $(\operatorname{DSDE})\left(\gamma, x_{0}\right)$ admit weak-strong existence and uniqueness.

(B) The marginal $\mu$-laws of the solution to (DSDE) $\left(\gamma, x_{0}\right)$ admit a density for $P \omega$ a.s.

(A) We start discussing well-posedness.

Proposition 4.1. We suppose the existence of random variables $A_{1}, A_{2}$ such that

$$
0<A_{1}(\omega) \leq \gamma(t, \xi, \omega) \leq A_{2}(\omega), \quad \forall(t, \xi) \in[0, T] \times \mathbb{R}, \quad \mathrm{d} P \text {-a.s. }
$$

Then $(D S D E)\left(\gamma, x_{0}\right)$ admits weak-strong existence and uniqueness.

Proof. Uniqueness. This is the easy part. Let $Y$ and $\widetilde{Y}$ be two solutions. Then for $\omega$ outside a $P$-null set $N_{0}, Y(\cdot, \omega)$ and $\widetilde{Y}(\cdot, \omega)$ are solutions to the same one-dimensional classical SDE with measurable bounded and non-degenerate (i.e. greater than a strictly positive constant) coefficients. Then, by Exercise 7.3.3 of [27] the law of $Y(\cdot, \omega)$ equals the law of $\widetilde{Y}(\cdot, \omega)$. Then obviously the law of $Y$ equals the law of $\widetilde{Y}$.

Existence. This point is more delicate. In fact one needs to solve the random SDE for $P$ almost all $\omega$ but in such a way that the solution produces bimeasurable processes $Y$ and $B$.

First we regularize the coefficient $\gamma$. Let $\phi$ be a mollifier with compact support; we set $\phi_{n}(x)=n \phi(n x), x \in \mathbb{R}$, $n \in \mathbb{N}$. We consider the random fields $\gamma_{n}:[0, T] \times \mathbb{R} \times \Omega \rightarrow \mathbb{R}$ by $\gamma_{n}(t, x, \omega):=\int_{\mathbb{R}} \gamma(t, x-y, \omega) \phi_{n}(y) \mathrm{d} y$.

Let $\left(\widetilde{\Omega}_{1}, \widetilde{\mathcal{H}}_{1}, \widetilde{P}\right)$ be a probability space where we can construct a random variable $Y_{0}$ distributed according to $x_{0}$ and an independent Brownian motion $B$.

In this way on $\left(\widetilde{\Omega}_{1} \times \Omega, \widetilde{\mathcal{H}}_{1} \otimes \mathcal{F}, \widetilde{P} \otimes P\right)$ we dispose of a random variable $Y_{0}$ and a Brownian motion independent of $\left\{\varnothing, \widetilde{\Omega}_{1}\right\} \otimes \mathcal{F}$. By usual fixed point techniques, it is possible to exhibit a (strong) solution of (DSDE) $\left(\gamma_{n}, x_{0}\right)$ on the over mentioned product probability space. We can show that there is a unique solution $Y=Y^{n}$ of $Y_{t}=Y_{0}+$ $\int_{0}^{t} \gamma_{n}\left(s, Y_{s}, \cdot\right) \mathrm{d} B_{s}$. In fact, the maps $\Gamma_{n}: Z \mapsto \int_{0}^{\cdot} \gamma_{n}\left(s, Z_{s}, \omega\right) \mathrm{d} B_{s}+Y_{0}$, where $\Gamma_{n}: L^{2}\left(\widetilde{\Omega}_{1} \times \Omega ; \widetilde{P} \otimes P\right) \rightarrow L^{2}\left(\widetilde{\Omega}_{1} \times\right.$ $\Omega, \widetilde{P} \otimes P$ ) are Lipschitz; by usual Picard fixed point arguments one can show the existence of a unique solution $Z=Z^{n}$ in $L^{2}\left(\widetilde{\Omega}_{1} \times \Omega ; \widetilde{P} \otimes P\right)$. We observe that, by usual regularization arguments for Itô integral as in Lemma 3.5, for $\omega$-a.s., $Y(\cdot, \omega)$ solves for $P$ a.e. $\omega \in \Omega$, equation

$$
Y_{t}(\cdot, \omega)=Y_{0}+\int_{0}^{t} \gamma_{n}\left(s, Y_{s}(\cdot, \omega), \omega\right) \mathrm{d} B_{s},
$$


on $\left(\widetilde{\Omega}_{1}, \widetilde{\mathcal{H}}_{1}, \widetilde{P}\right)$. We consider now the measurable space $\Omega_{0}=\Omega_{1} \times \Omega$, where $\Omega_{1}=C([0, T], \mathbb{R})$, equipped with product $\sigma$-field $\mathcal{G}=\mathcal{B}\left(\Omega_{1}\right) \otimes \mathcal{F}$. On that measurable space, we introduce the probability measures $\mathbf{Q}_{n}$ where $\mathbf{Q}_{n}\left(\mathrm{~d} \omega_{1}, \omega\right)=Q_{n}\left(\mathrm{~d} \omega_{1}, \omega\right) P(\mathrm{~d} \omega)$ and $Q_{n}(\cdot, \omega)$ being the law of $Y^{n}(\cdot, \omega)$ for almost all fixed $\omega$.

We set $Y_{t}\left(\omega_{1}, \omega\right)=\omega_{1}(t)$, where $\omega_{1} \in C([0, T] ; \mathbb{R})$. We denote by $\left(\mathcal{Y}_{t}, t \in[0, T]\right)$ (resp. $\left.\left(\mathcal{Y}_{t}^{1}\right)\right)$ the canonical filtration associated with $Y$ on $\Omega_{0}$ (resp. $\Omega_{1}$ ). The next step will be the following.

Lemma 4.2. For almost all $\omega d P$ a.s. $Q_{n}(\omega, \cdot)$ converges weakly to $Q(\omega, \cdot)$, where under $Q(\cdot, \omega), Y(\cdot, \omega)$ solves the $S D E$

$$
Y_{t}(\cdot, \omega)=Y_{0}+\int_{0}^{t} \gamma\left(s, Y_{s}(\cdot, \omega), \omega\right) \mathrm{d} B_{s}(\cdot, \omega)
$$

where $B(\cdot, \omega)$ is an $\left(\mathcal{Y}_{t}^{1}\right)$-Brownian motion on $\Omega_{1}$.

Proof. It follows directly from Proposition A.1 of the Appendix.

This shows the validity of (1) if Definition 2.10(a).

\section{Remark 4.3.}

(1) Since $Q_{n}(\cdot, \omega)$ converges weakly to $Q(\cdot, \omega), \omega d P$ a.s., then the limit (up to an obvious modification) is a measurable random kernel.

(2) This also implies that $Y_{n}(\cdot, \omega)$ converges stably to $Q(\cdot, \omega)$. For details about the stable convergence the reader can consult [17, section VIII 5.c] and the recent monograph [16].

The considerations above allow to complete the proof of Proposition 4.1. By Lemma 4.2, $Q^{\omega}=Q(\cdot, \omega)$ is a random kernel, being a limit of random kernels. Let us consider the associated probability measure on the suitable enlarged probability space $\left(\Omega_{0}, \mathcal{G}, Q\right)$. We observe that $Y$ on $\left(\Omega_{0}, \mathcal{G}\right)$ is obviously measurable, because it is the canonical process $Y\left(\omega_{1}, \omega\right)=\omega_{1}$. Setting

$$
B_{t}(\cdot, \omega)=\int_{0}^{t} \frac{\mathrm{d} Y_{s}(\cdot, \omega)}{\gamma\left(s, Y_{S}(\cdot, \omega), \omega\right)}
$$

we get $[B]_{t}(\cdot, \omega)=t$ under $Q(\cdot, \omega)$, so, by Lévy characterization theorem, it is a Brownian motion. Moreover $B$ is bimeasurable.

Let $G=\mathcal{A}\left(Y_{r}(\cdot, \omega), r \in[0, s]\right)$, where $\mathcal{A}$ is a bounded functional $C([0, s]) \rightarrow \mathbb{R}$. We first observe that the r.v. $\omega \mapsto E^{Q^{\omega}}(G)$ is $\mathcal{F}_{s}$-measurable. This happens because $Y$ is, under $Q^{\omega}$, a martingale with quadratic variation $\left(\int_{0}^{t} \gamma^{2}\left(s, Y_{s}(\cdot, \omega), \omega\right) \mathrm{d} s, 0 \leq t \leq T\right)$, i.e. with (random) coefficient which is $\left(\mathcal{F}_{t}\right)$-progressively measurable. This shows item (3) of Definition 2.10(a).

The last point to check is that $W^{1}, \ldots, W^{N}$ are $\left(\mathcal{G}_{t}\right)$-martingales, where $\mathcal{G}_{t}=\mathcal{Y}_{t} \vee\left(\left\{\varnothing, \Omega_{1}\right\} \otimes \mathcal{F}_{t}\right), 0 \leq t \leq T$, i.e. item (2) of Definition 2.10.

Indeed, we justify this immediately. Consider $0 \leq s \leq t \leq T$. Taking into account monotone class arguments, given $F \in \mathcal{F}_{s}, G \in \mathcal{Y}_{s}^{1}, 1 \leq i \leq N$, it is enough to prove that

$$
E^{\mathbf{Q}}\left(F G W_{t}^{i}\right)=E^{\mathbf{Q}}\left(F G W_{s}^{i}\right)
$$

Using the fact that $W^{i}$ is an $\left(\mathcal{F}_{t}\right)$-martingale and that $E^{Q^{\omega}}(G)$ is $\mathcal{F}_{s}$-measurable by item (a)(3) of Definition 2.10 (established above), the left-hand side of (4.2) gives

$$
E^{P}\left(F W_{t}^{i} E^{Q^{\omega}}(G)\right)=E^{P}\left(F W_{s}^{i} E^{Q^{\omega}}(G)\right),
$$

which constitutes the right-hand side of (4.2). This concludes the proof of the proposition.

We go on now with step (B) of the beginning of Section 4. 
Proposition 4.4. We suppose the existence of r.v. $A_{1}, A_{2}$ such that

$$
0<A_{1}(\omega) \leq \gamma(t, \xi, \omega) \leq A_{2}(\omega), \quad \forall(t, \xi) \in[0, T] \times \mathbb{R}, \quad \text { a.s. }
$$

Let $Y$ be a weak-strong solution to $(D S D E)\left(\gamma, x_{0}\right)$ and we denote by $\left(v_{t}(d y, \cdot), t \in[0, T]\right)$, the $\mu$-marginal weighted laws of process $Y$.

1. There is a measurable function $q:[0, T] \times \mathbb{R} \times \Omega \rightarrow \mathbb{R}_{+}$such that $\mathrm{d} t \mathrm{~d} P$ a.e., $v_{t}(\mathrm{~d} y, \cdot)=q_{t}(y, \cdot) \mathrm{d} y$. In other words the $\mu$-marginal weighted laws admit densities.

2. $\int_{[0, T] \times \mathbb{R}} q_{t}^{2}(y, \cdot) \mathrm{d} t \mathrm{~d} y<\infty \mathrm{d} P$-a.s.

3. $q$ is an $L^{2}(\mathbb{R})$-valued progressively measurable process.

Proof. By (3) of Definition 2.10, the $\mu$-marginal laws constitute an $\mathcal{S}^{\prime}(\mathbb{R})$-valued progressively measurable process. Consequently 3 . holds if 1 . and 2 . hold.

Let

$$
B_{t}(\cdot, \omega):=\int_{0}^{t} \frac{\mathrm{d} Y_{s}(\cdot, \omega)}{\gamma\left(s, Y_{s}(\cdot, \omega), \omega\right)} .
$$

We denote again $Q^{\omega}:=Q(\cdot, \omega)$ according to Definition 2.10, $\omega \in \Omega$.

Let $\omega \in \Omega$ be fixed. Let $\varphi:[0, T] \times \mathbb{R} \rightarrow \mathbb{R}$ be a continuous function with compact support. We need to evaluate

$$
E^{Q^{\omega}}\left(\int_{0}^{T} \varphi\left(s, Y_{s}\right) Z_{s} \mathrm{~d} s\right)
$$

where $Z_{s}=M_{s} \exp \left(\int_{0}^{s} e^{0}\left(Y_{r}\right) \mathrm{d} r\right)$ where $M_{s}=\mathcal{E}_{s}\left(\sum_{i=1}^{N} \int_{0}^{\cdot} e^{i}\left(Y_{r}\right) \mathrm{d} W_{r}^{i}\right)$.

$M_{s}$ is smaller or equal than $\exp \left(\sum_{j=1}^{N} \int_{0}^{s} e^{j}\left(Y_{r}\right) \mathrm{d} W_{r}^{j}\right)$ which equals

$$
\exp \left(\sum_{j=1}^{N}\left\{W_{s}^{j} e^{j}\left(Y_{s}\right)-\int_{0}^{s} W_{r}^{j}\left(e^{j}\right)^{\prime}\left(Y_{r}\right) \mathrm{d} Y_{r}\right\}-\frac{1}{2} \int_{0}^{s}\left\{\sum_{j=1}^{N} W_{r}^{j}\left(e^{j}\right)^{\prime \prime}\left(Y_{r}\right) \gamma^{2}\left(r, Y_{r}, \cdot\right)\right\} \mathrm{d} r\right),
$$

taking into account the fact that $\left[Y, W^{j}\right]=0$ for any $1 \leq j \leq n$, by Proposition 2.12. Denoting $\|g\|_{\infty}:=$ $\sup _{t \in[0, T]}|g(t)|$, for a function $g:[0, T] \rightarrow \mathbb{R},(4.4)$ is smaller or equal than

$$
\exp \left(\sum_{j=1}^{N}\left\|W^{j}\right\|_{\infty}\left(\left\|e^{j}\right\|_{\infty}+\frac{T}{2}\left\|\left(e^{j}\right)^{\prime \prime}\right\|_{\infty} A_{2}^{2}\right)\right) \exp \left(-\int_{0}^{s}\left[\sum_{j=1}^{N} W_{r}^{j}\left(e^{j}\right)^{\prime}\left(Y_{r}\right) \gamma\left(r, Y_{r}, \cdot\right)\right] \mathrm{d} B_{r}\right) .
$$

So (4.3) is bounded by

$$
\varrho(\omega) E^{Q^{\omega}}\left(\int_{0}^{T}|\varphi|\left(s, Y_{S}(\cdot, \omega)\right) R_{S}(\cdot, \omega) \mathrm{d} s\right)
$$

where

$$
\begin{aligned}
\varrho(\omega)= & \exp \left(T\left\|e_{0}\right\|_{\infty}+\sum_{i=1}^{N}\left\|W^{i}\right\|_{\infty}\left\|e^{i}\right\|_{\infty}\right. \\
& \left.+T \frac{A_{2}^{2}(\omega)}{2} \sum_{i=1}^{N}\left(\left\|W^{i}\right\|_{\infty}^{2}\left\|\left(e^{i}\right)^{\prime}\right\|_{\infty}^{2}+\left\|W^{i}\right\|_{\infty}\left\|\left(e^{i}\right)^{\prime \prime}\right\|_{\infty}\right)\right)
\end{aligned}
$$

and $R$ is the $Q^{\omega}$-exponential martingale

$$
R_{t}(\cdot, \omega)=\exp \left(-\int_{0}^{t} \delta(r, \cdot, \omega) \mathrm{d} B_{r}-\frac{1}{2} \int_{0}^{t} \delta^{2}(r, \cdot, \omega) \mathrm{d} r\right)
$$


where $\delta(r, \cdot, \omega)=\sum_{j=1}^{N} W_{r}^{j}\left(e^{j}\right)^{\prime}\left(Y_{r}(\cdot, \omega)\right) \gamma\left(r, Y_{r}(\cdot, \omega), \omega\right)$. So there is a random (depending on $\left.\omega \in \Omega\right)$ constant

$$
\varrho_{1}(\omega):=\operatorname{const}\left(T, W^{j},\left\|e^{j}\right\|_{\infty},\left\|\left(e^{j}\right)^{\prime}\right\|_{\infty},\left\|\left(e^{j}\right)^{\prime \prime}\right\|_{\infty}, 1 \leq j \leq N, A_{2}(\omega)\right),
$$

so that (4.5) is smaller than

$$
\varrho_{1}(\omega) E^{Q^{\omega}}\left(\int_{0}^{T}\left|\varphi\left(s, Y_{s}(\cdot, \omega)\right)\right| \mathrm{d} s R_{T}(\cdot, \omega)\right),
$$

where we remind that $R(\cdot, \omega)$ is a $Q^{\omega}$-martingale. By Girsanov theorem, $\widetilde{B}_{t}(\cdot, \omega)=B_{t}(\cdot, \omega)+\int_{0}^{t} \delta(r, \cdot, \omega) \mathrm{d} r$ is a $\widetilde{Q}^{\omega}$-Brownian motion with $\mathrm{d} \widetilde{Q}^{\omega}=R_{T}(\cdot, \omega) \mathrm{d} Q^{\omega}$. At this point, the expectation in (4.7) gives

$$
E^{\widetilde{Q}^{\omega}}\left(\int_{0}^{T}|\varphi|\left(s, Y_{s}(\cdot, \omega)\right) \mathrm{d} s\right)
$$

where

$$
Y_{t}(\cdot, \omega)=Y_{0}+\int_{0}^{t} \gamma\left(s, Y_{s}(\cdot, \omega), \omega\right) \mathrm{d} \widetilde{B}_{s}-\int_{0}^{t} \gamma\left(s, Y_{s}(\cdot, \omega), \omega\right) \delta(s, \cdot, \omega) \mathrm{d} s .
$$

For fixed $\omega \in \Omega, \delta$ is bounded by a random constant $\varrho_{2}(\omega)$ of the type (4.6). Moreover we keep in mind assumption (4.1) on $\gamma$. By Exercise 7.3.3 of [27], (4.8) is bounded by $\varrho_{3}(\omega)\|\varphi\|_{L^{2}([0, T] \times \mathbb{R})}$, where $\varrho_{3}(\omega)$ again depends on the same quantities as in (4.6) and $\Phi$. So for $\omega \mathrm{d} P$-a.s., the map $\varphi \mapsto E^{Q^{\omega}}\left(\int_{0}^{T} \varphi\left(s, Y_{S}(\cdot, \omega)\right) Z_{S}(\cdot, \omega) \mathrm{d} s\right)$ prolongs to $L^{2}([0, T] \times \mathbb{R})$. Using Riesz' theorem it is not difficult to show the existence of an $L^{2}([0, T] \times \mathbb{R})$ function $(s, y) \mapsto q_{s}(y, \omega)$ which constitutes indeed the density of the family of the $\mu$-marginal weighted laws.

\section{On the uniqueness of a Fokker-Planck type SPDE}

The next result is an extension of Theorem 3.8 of [14] to the stochastic case. It has an independent interest since it is a Fokker-Planck SPDE with possibly degenerate measurable coefficients.

Theorem 5.1. Let $z_{0}$ be a distribution in $\mathcal{S}^{\prime}(\mathbb{R})$. Let $z^{1}, z^{2}$ be two measurable random fields belonging $\omega$ a.s. to $C\left([0, T], \mathcal{S}^{\prime}(\mathbb{R})\right)$ such that $\left.\left.z^{1}, z^{2}:\right] 0, T\right] \times \Omega \rightarrow \mathcal{M}(\mathbb{R})$. Let $a:[0, T] \times \mathbb{R} \times \Omega \rightarrow \mathbb{R}_{+}$be a bounded measurable random field such that, for any $t \in[0, T], a(t, \cdot)$ is $\mathcal{B}([0, t]) \otimes \mathcal{B}(\mathbb{R}) \otimes \mathcal{F}_{t}$-measurable. We suppose moreover the following.

(i) $z^{1}-z^{2} \in L^{2}([0, T] \times \mathbb{R})$ a.s.

(ii) $t \mapsto\left(z^{1}-z^{2}\right)(t, \cdot)$ is an $\left(\mathcal{F}_{t}\right)$-progressively measurable $\mathcal{S}^{\prime}(\mathbb{R})$-valued process.

(iii) $\int_{0}^{T}\left\|z^{i}(s, \cdot)\right\|_{\text {var }}^{2} \mathrm{~d} s<\infty$ a.s.

(iv) $z^{1}, z^{2}$ are solutions to

$$
\left\{\begin{array}{l}
\partial_{t} z(t, \xi)=\partial_{\xi \xi}^{2}((a z)(t, \xi))+z(t, \xi) \mu(\mathrm{d} t, \xi) \\
z(0, \cdot)=z_{0}
\end{array}\right.
$$

Then $z^{1} \equiv z^{2}$.

Remark 5.2. By solution of equation (5.1) we intend, as expected, the following: for every $\varphi \in \mathcal{S}(\mathbb{R}), \forall t \in[0, T]$,

$$
\int_{\mathbb{R}} \varphi(\xi) z(t, \mathrm{~d} \xi)=\left\langle z_{0}, \varphi\right\rangle+\int_{0}^{t} \mathrm{~d} s \int_{\mathbb{R}} a(s, \xi) \varphi^{\prime \prime}(\xi) z(s, \mathrm{~d} \xi)+\sum_{j=0}^{N} \int_{0}^{t} \mathrm{~d} W_{s}^{j} \int_{\mathbb{R}} \varphi(\xi) e^{j}(\xi) z(s, d \xi) .
$$

Proof of Theorem 5.1. The proof makes use of the similar arguments as in Theorem 3.8 of [14] or Theorem 3.1 in [10], in a randomized form. The full proof is given in Theorem 3.1 of [24], see also Theorem 5.1 of [7]. 


\section{The non-degenerate case}

We are now able to discuss the doubly probabilistic representation of a solution to (1.1) when $\psi$ is non-degenerate provided that its solution fulfills some properties.

\section{Definition 6.1.}

- We will say that equation (1.1) (or $\psi$ ) is non-degenerate if on each compact, there is a constant $c_{0}>0$ such that $\Phi \geq c_{0}$.

- We will say that equation (1.1) or $\psi$ is degenerate if $\lim _{u \rightarrow 0_{+}} \Phi(u)=0$.

One of the typical examples of degenerate $\psi$ is the case of $\psi$ being strictly increasing after some zero. This notion was introduced in [6] and it means the following. There is $0 \leq u_{c}$ such that $\psi_{\left[0, u_{c}\right]} \equiv 0$ and $\psi$ is strictly increasing on ]$u_{c},+\infty[$.

\section{Remark 6.2.}

1. $\psi$ is non-degenerate if and only if $=\lim _{u \rightarrow 0+} \Phi(u)>0$.

2. Of course, if $\psi$ is strictly increasing after some zero, with $u_{c}>0$ then $\psi$ is degenerate.

3. If $\psi$ is degenerate, then $\psi^{\kappa}(u)=\left(\Phi^{2}(u)+\kappa\right) u$, for every $\kappa>0$, is non-degenerate.

As announced the theorem below also holds when $\psi$ is multi-valued.

Theorem 6.3. We suppose the following assumptions.

1. $x_{0}$ is a real probability measure.

2. $\psi$ is non-degenerate.

3. There is only one random field $X:[0, T] \times \mathbb{R} \times \Omega \rightarrow \mathbb{R}$ solution of (1.1) (see Definition 2.9) such that

$$
\int_{[0, T] \times \mathbb{R}} X^{2}(s, \xi) \mathrm{d} s \mathrm{~d} \xi<\infty \quad \text { a.s. }
$$

Then there is a unique weak solution to the $(D S N L D)\left(\Phi, \mu, x_{0}\right)$.

\section{Remark 6.4.}

1. An easy adaptation of Theorem 3.4 of [8] (taking into account $e^{0}$ ), when $\psi$ is Lipschitz and $e^{0}, \ldots, e^{N}$ belong to $H^{1}$ allows to show that there is a solution to (1.1) such that $E\left(\int_{[0, T] \times \mathbb{R}} X^{2}(s, \xi) \mathrm{d} s \mathrm{~d} \xi\right)<\infty$. This holds even if $x_{0}$ belongs to $H^{-1}(\mathbb{R})$. According to Theorem B.1, that solution is unique. In particular item 3 . in Theorem 6.3 statement holds.

2. Theorem 6.3 constitutes the converse of Theorem 3.3 when $\psi$ is non-degenerate.

3. The theorem also holds if $\psi$ is multi-valued. For implementing this, we need to adapt the techniques of [14].

4. As side-effect of the proof of the weak-strong existence Proposition 4.1, the space $\left(\Omega_{0}, \mathcal{G}, \mathbf{Q}\right)$ can be chosen as $\Omega_{0}=\Omega_{1} \times \Omega, \Omega_{1}=C([0, T] ; \mathbb{R}) \times \mathbb{R}, \mathcal{G}=\mathcal{B}\left(\Omega_{1}\right) \times \mathcal{F}, \mathbf{Q}(H \times F)=\int_{\Omega_{1} \times \Omega} \mathrm{d} P(\omega) 1_{F}(\omega) Q\left(\mathrm{~d} \omega_{1}, \omega\right)$.

\section{Proof of Theorem 6.3.}

(1) We set $\gamma(t, \xi, \omega)=\Phi(X(t, \xi, \omega))$. According to Proposition 4.1 there is a weak-strong solution $Y$ to (DSDE) $\left(\gamma, x_{0}\right)$. By Proposition $4.4 \omega$ a.s. the $\mu$-marginal weighted laws of $Y$ admit densities $\left(q_{t}(\xi, \omega), t \in\right.$ ] $0, T], \xi \in \mathbb{R}, \omega \in \Omega$ ) such that $\mathrm{d} P$-a.s. $\int_{[0, T] \times \mathbb{R}} \mathrm{d} s \mathrm{~d} \xi q_{s}^{2}(\xi, \cdot)<\infty$ a.s.

(2) Setting

$$
v_{t}(\xi, \omega)= \begin{cases}q_{t}(\xi, \omega) \mathrm{d} \xi, & t \in] 0, T], \\ x_{0}, & t=0,\end{cases}
$$


$v$ is a solution to (5.1) with $v_{0}=x_{0}, a(t, \xi, \omega)=\Phi^{2}(X(t, \xi, \omega))$. This can be shown applying Itô's formula similarly as in the proof of Theorem 3.3.

(3) On the other hand $X$ is obviously also a solution of (5.1), which in particular verifies (6.1). Consequently $z^{1}=$ $v, z^{2}=X$ verify items (i), (ii), (iii) of Theorem 5.1. So Theorem 5.1 implies that $v \equiv X$; this shows that $Y$ provides a solution to $(\mathrm{DSNLD})\left(\Phi, \mu, x_{0}\right)$.

(4) Concerning uniqueness, let $Y^{1}, Y^{2}$ be two solutions to the (DSNLD) related to $(\Phi, \mu, x)$. The corresponding random fields $X^{1}, X^{2}$ constitute the $\mu$-marginal laws of $Y^{1}, Y^{2}$ respectively.

Now $Y^{i}, i=1,2$, is a weak-strong solution of (DSDE) $\left(\gamma_{i}, x\right)$ with $\gamma_{i}(t, \xi, \omega)=\Phi\left(X_{i}(t, \xi, \omega)\right)$, so by Proposition 4.4 $X_{i}, i=1,2$ fulfills (6.1). By Theorem 3.3, $X_{1}$ and $X_{2}$ are solutions to (1.1). By assumption 3. of the statement, $X_{1}=X_{2}$. The conclusion follows by Proposition 4.1, which guarantees the uniqueness of the weak-strong solution of $(\mathrm{DSDE})\left(\gamma, x_{0}\right)$ with $\gamma=\gamma_{1}=\gamma_{2}$.

Remark 6.5. One side-effect of Theorem 6.3 is the following. Suppose $\psi$ to be non-degenerate. Let $X:[0, T] \times \mathbb{R} \times$ $\Omega \rightarrow \mathbb{R}$ be a solution such that $\mathrm{d} P$-a.s. $\int_{[0, T] \times \mathbb{R}} X^{2}(s, \xi) \mathrm{d} s \mathrm{~d} \xi<\infty$ a.s. We have the following for $\omega \mathrm{d} P$-a.s.

(i) $X(t, \cdot, \omega) \geq 0$ a.e. $\forall t \in[0, T]$.

(ii) $E\left(\int_{\mathbb{R}} X(t, \xi) \mathrm{d} \xi\right)=1, \forall t \in[0, T]$ if $e_{0}=0$.

Remark 6.6. If (1.1) has a solution, not necessarily unique, then (DSNLD) with respect to $\left(\Phi, \mu, x_{0}\right)$ still admits existence.

\section{The degenerate case}

The idea consists in proceeding similarly to [6], which treated the case $\mu=0$ and the case when $x_{0}$ is absolutely continuous with bounded density. $\psi$ will be assumed to be strictly increasing after some zero $u_{c} \geq 0$, see Definition 6.1. We recall that if $\psi$ is degenerate, then necessarily $\Phi(0):=\lim _{x \rightarrow 0} \Phi(x)=0$.

The theorem below concerns existence, we do not know any uniqueness result in the degenerate case.

Theorem 7.1. We suppose the following.

1. The functions $e^{i}, 1 \leq i \leq N$ belong to $H^{1}(\mathbb{R})$.

2. We suppose that $\psi: \mathbb{R} \rightarrow \mathbb{R}$ is non-decreasing, Lipschitz and strictly increasing after some zero.

3. $x_{0}$ belongs to $L^{1}(\mathbb{R}) \cap L^{2}(\mathbb{R})$.

Then there is a weak solution to the $(D S N L D)\left(\Phi, \mu, x_{0}\right)$.

Remark 7.2. If $u_{c}>0$ then $\psi$ is necessarily degenerate and also $\Phi$ restricted to $\left[0, u_{c}\right]$ vanishes.

\section{Proof of Theorem 7.1.}

(1) We proceed by approximation rendering $\Phi$ non-degenerate. Let $\kappa>0$. We define $\Phi_{\kappa}: \mathbb{R} \rightarrow \mathbb{R}_{+}$by $\Phi_{\kappa}(u)=$ $\sqrt{\Phi^{2}(u)+\kappa}, \psi_{\kappa}(u)=\Phi_{\kappa}^{2}(u) \cdot u$. Let $X^{\kappa}$ be the solution so (1.1) with $\psi_{\kappa}$ instead of $\psi$. According to Theorem 6.3 and Remark 6.4 4., setting

$$
\widetilde{\Omega}_{1}=C([0, T], \mathbb{R}) \times \mathbb{R}, \quad Y\left(\omega_{1}, \omega\right)=\omega_{1},
$$

$\mathcal{H}$ the Borel $\sigma$-algebra of $\widetilde{\Omega}_{1}$, there are families of probability kernels $Q^{\kappa}$ on $\mathcal{H} \times \Omega$, and measurable processes $B^{\kappa}$ on $\widetilde{\Omega}_{0}=\widetilde{\Omega}_{1} \times \Omega$ such that

(i) $B^{\kappa}(\cdot, \omega)$ is a $Q^{\kappa}(\cdot, \omega)$-Brownian motion;

(ii) $Y$ is a (weak) solution, on $\left(\tilde{\Omega}_{1}, Q^{\kappa}(\cdot, \omega)\right)$, of $Y_{t}=Y_{0}+\int_{0}^{t} \Phi_{\kappa}\left(X^{\kappa}\left(s, Y_{s}, \omega\right)\right) \mathrm{d} B_{s}^{\kappa}(\cdot, \omega), t \in[0, T]$;

(iii) $Y_{0}$ is distributed according to $x_{0}=X^{\kappa}(0, \cdot)$;

(iv) The $\mu$-marginal weighted laws of $Y$ under $\mathbf{Q}^{\kappa}$ are $\left(X^{\kappa}(t, \cdot)\right)$.

In agreement with Definition 3.1 and Definition 2.10, we need to show the existence of a suitable measurable space $\left(\Omega_{1}, \mathcal{H}\right)$, a probability kernel $Q$ on $\mathcal{H} \times \Omega$, a process $B$ on $\Omega_{0}:=\Omega_{1} \times \Omega$ such that the following holds. 
(i) $B(\cdot, \omega)$ is a $Q(\cdot, \omega)$-Brownian motion.

(ii) $Y$ is a (weak) solution on $\left(\Omega_{1}, Q(\cdot, \omega)\right)$ of $Y_{t}=Y_{0}+\int_{0}^{t} \Phi\left(X\left(s, Y_{s}, \omega\right)\right) \mathrm{d} B_{s}(\cdot, \omega), t \in[0, T]$, i.e. item (1) of Definition 2.10. Moreover items (2), (3) of the same Definition are fulfilled.

(iii) $Y_{0}$ is distributed according to $x_{0}$.

(iv) For every $t \in] 0, T], \varphi \in C_{b}(\mathbb{R})$, if we denote $Q^{\omega}=Q(\cdot, \omega)$, we have

$$
\int_{\mathbb{R}} X(t, \xi) \varphi(\xi) \mathrm{d} \xi=E^{Q^{\omega}}\left(\varphi\left(Y_{t}\right) \mathcal{E}_{t}\left(\int_{0}^{\cdot} \mu\left(\mathrm{d} s, Y_{s}\right) X\left(s, Y_{s}\right)\right)\right) .
$$

(2) We show now that $X^{\kappa}$ approaches $X$ in some sense when $\kappa \rightarrow 0$, where $X$ is the solution to (1.1). This is given in the Lemma 7.3 below.

Lemma 7.3. Under the assumptions of Theorem 7.1, according to Remark B.2, let X (resp. $X^{\kappa}$ ) be a solution of (1.1) verifying (B.2) with $\psi(u)=u \Phi^{2}(u)$ (resp. $\psi_{\kappa}(u)=u\left(\Phi^{2}(u)+\kappa\right)$ ), for $u>0$. We have the following.

(a) $\lim _{\kappa \rightarrow 0} \sup _{t \in[0, T]} E\left(\left\|X^{\kappa}(t, \cdot)-X(t, \cdot)\right\|_{H^{-1}}^{2}\right)=0$;

(b) $\lim _{\kappa \rightarrow 0} E\left(\int_{0}^{T} \mathrm{~d} t\left\|\psi\left(X^{\kappa}(t, \cdot)\right)-\psi(X(t, \cdot))\right\|_{L^{2}}^{2}\right)=0$;

(c) $\lim _{\kappa \rightarrow 0} \kappa E\left(\int_{[0, T] \times \mathbb{R}} \mathrm{d} t \mathrm{~d} \xi\left(X^{\kappa}(t, \xi)-X(t, \xi)\right)^{2}\right)=0$.

\section{Remark 7.4.}

(1) (a) implies of course $\lim _{\kappa \rightarrow 0} E\left(\int_{0}^{T} \mathrm{~d} t\left\|X^{\kappa}(t, \cdot)-X(t, \cdot)\right\|_{H^{-1}}^{2}\right)=0$.

(2) In particular Lemma 7.3(b) implies that for each sequence $\left(\kappa_{n}\right) \rightarrow 0$ there is a subsequence, still denoted by the same notation, that $\int_{[0, T] \times \mathbb{R}}\left(\psi\left(X^{\kappa_{n}}(t, \xi)\right)-\psi(X(t, \xi))\right)^{2} \mathrm{~d} t \mathrm{~d} \xi \rightarrow_{n \rightarrow \infty} 0$ a.s.

(3) For every $t \in[0, T] X(t, \cdot) \geq 0 d \xi \otimes d P$ a.e. Indeed, for this it will be enough to show that a.s.

$$
\int_{\mathbb{R}} \mathrm{d} \xi \varphi(\xi) X(t, \xi) \geq 0 \quad \text { for every } \varphi \in \mathcal{S}(\mathbb{R}),
$$

for every $t \in[0, T]$. Since $X \in C\left([0, T] ; \mathcal{S}^{\prime}(\mathbb{R})\right)$ it will be enough to show (7.2) for almost all $t \in[0, T]$. This holds true since item (1) in this Remark 7.4, implies the existence of a sequence $\left(\kappa_{n}\right)$ such that $\int_{0}^{t} \mathrm{~d} t \| X^{\kappa_{n}}(t, \cdot)-$ $X(t, \cdot) \|_{H^{-1}}^{2} \rightarrow_{n \rightarrow \infty} 0$, a.s.

(4) Since $\psi$ is strictly increasing after $u_{c}$, then, for $P$ almost all $\omega$, for almost all $(t, \xi) \in[0, T] \times \mathbb{R}$, there is a sequence $\left(\kappa_{n}\right)$ such that $\left(X^{\kappa_{n}}(t, \xi)-X(t, \xi)\right) 1_{\left\{X(t, \xi)>u_{c}\right\}} \rightarrow_{n \rightarrow \infty} 0$. This follows from item (2) of Remark 7.4.

Since $\Phi^{2}(u)=0$ for $0 \leq u \leq u_{c}$ and $X$ is a.e. non-negative, this implies that $d t d \xi d P$ a.e. we have

$$
\Phi^{2}(X(t, \xi))\left(X^{\kappa_{n}}(t, \xi)-X(t, \xi)\right) \underset{n \rightarrow \infty}{\rightarrow} 0 .
$$

Proof of Lemma 7.3. By Remark B.2(3) we can write $\mathrm{d} P$-a.s. the following $H^{-1}(\mathbb{R})$-valued equality.

$$
\left(X^{\kappa}-X\right)(t, \cdot)=\int_{0}^{t} \mathrm{~d} s\left(\psi_{\kappa}\left(X^{\kappa}(s, \cdot)\right)-\psi(X(s, \cdot))\right)^{\prime \prime}+\sum_{i=0}^{N} \int_{0}^{t}\left(X^{\kappa}(s, \cdot)-X(s, \cdot)\right) e^{i} \mathrm{~d} W_{s}^{i} .
$$

So

$$
\begin{aligned}
(I-\Delta)^{-1}\left(X^{\kappa}-X\right)(t, \cdot)= & -\int_{0}^{t} \mathrm{~d} s\left(\psi_{\kappa}\left(X^{\kappa}(s, \cdot)\right)-\psi(X(s, \cdot))\right) \\
& +\int_{0}^{t} \mathrm{~d} s(I-\Delta)^{-1}\left(\psi_{\kappa}\left(X^{\kappa}(s, \cdot)\right)-\psi(X(s, \cdot))\right) \\
& +\sum_{i=0}^{N} \int_{0}^{t}(I-\Delta)^{-1}\left(e^{i}\left(X^{\kappa}(s, \cdot)-X(s, \cdot)\right)\right) \mathrm{d} W_{s}^{i} .
\end{aligned}
$$


After regularization and application of Itô calculus with values in $H^{-1}$, we will be able to estimate $g^{\kappa}(t)=\|\left(X^{\kappa}-\right.$ $X)(t, \cdot) \|_{H^{-1}}^{2}$. Taking advantage of the form of $\psi_{\kappa}-\psi$, we obtain

$$
\begin{aligned}
g^{\kappa}(t)= & \sum_{i=1}^{N} \int_{0}^{t}\left\|e^{i}\left(X^{\kappa}-X\right)(s, \cdot)\right\|_{H^{-1}}^{2} \mathrm{~d} s \\
& -2 \int_{0}^{t}\left\langle\left(X^{\kappa}-X\right)(s, \cdot), \psi_{\kappa}\left(X^{\kappa}(s, \cdot)\right)-\psi(X(s, \cdot))\right\rangle_{L^{2}} \\
& +2 \int_{0}^{t} \mathrm{~d} s\left\langle\left(X^{\kappa}-X\right)(s, \cdot),(I-\Delta)^{-1}\left(\psi_{\kappa}\left(X^{\kappa}(s, \cdot)\right)-\psi(X(s, \cdot))\right)\right\rangle_{L^{2}} \\
& +2 \int_{0}^{t} \mathrm{~d} s\left\langle\left(X^{\kappa}-X\right)(s, \cdot),(I-\Delta)^{-1} e^{0}\left(X^{\kappa}-X\right)(s, \cdot)\right\rangle_{L^{2}}+M_{t}^{\kappa},
\end{aligned}
$$

where $M^{\kappa}$ is the local martingale

$$
M_{t}^{\kappa}=2 \sum_{i=1}^{N} \int_{0}^{t}\left\langle(I-\Delta)^{-1}\left(X^{\kappa}-X\right)(s, \cdot),\left(X^{\kappa}-X\right)(s, \cdot) e^{i}\right\rangle_{L^{2}} \mathrm{~d} W_{s}^{i} .
$$

Indeed, $M^{\kappa}$ is a well-defined local martingale because, taking into account (B.1) and Remark B.2, using classical arguments, we can prove that

$$
\sum_{i=1}^{N} \int_{0}^{t}\left|\left\langle\left(X^{\kappa}-X\right)(s, \cdot),(I-\Delta)^{-1}\left(X^{\kappa}-X\right)(s, \cdot) e^{i}\right\rangle_{L^{2}}\right|^{2} \mathrm{~d} s<\infty \quad \text { a.s. }
$$

(7.4) gives

$$
\begin{aligned}
g^{\kappa}(t) & +2 \int_{0}^{t}\left\langle\left(X^{\kappa}-X\right)(s, \cdot), \psi\left(X^{\kappa}(s, \cdot)\right)-\psi(X(s, \cdot))\right\rangle_{L^{2}} \mathrm{~d} s \\
& +2 \kappa \int_{0}^{t}\left\langle\left(X^{\kappa}-X\right)(s, \cdot),\left(X^{\kappa}-X\right)(s, \cdot)\right\rangle_{L^{2}} \mathrm{~d} s \\
\leq & -2 \kappa \int_{0}^{t} \mathrm{~d} s\left\langle\left(X^{\kappa}-X\right)(s, \cdot), X(s, \cdot)\right\rangle_{L^{2}} \mathrm{~d} s+\sum_{i=1}^{N} \int_{0}^{t}\left\|e^{i}\left(X^{\kappa}-X\right)(s, \cdot)\right\|_{H^{-1}}^{2} \mathrm{~d} s \\
& +2 \int_{0}^{t} \mathrm{~d} s\left\langle(I-\Delta)^{-1}\left(X^{\kappa}-X\right)(s, \cdot), \psi\left(X^{\kappa}(s, \cdot)\right)-\psi(X(s, \cdot))\right\rangle_{L^{2}} \\
& +2 \kappa \int_{0}^{t} \mathrm{~d} s\left\langle(I-\Delta)^{-1}\left(X^{\kappa}-X\right)(s, \cdot),\left(X^{\kappa}-X\right)(s, \cdot)\right\rangle_{L^{2}} \\
& +2 \kappa \int_{0}^{t} \mathrm{~d} s\left\langle(I-\Delta)^{-1}\left(X^{\kappa}-X\right)(s, \cdot), X(s, \cdot)\right\rangle_{L^{2}} \\
& +2 \int_{0}^{t} \mathrm{~d} s\left\langle(I-\Delta)^{-1}\left(X^{\kappa}-X\right)(s, \cdot),\left(e^{0}\left(X^{\kappa}-X\right)(s, \cdot)\right)\right\rangle_{L^{2}}+M_{t}^{\kappa} .
\end{aligned}
$$

We use Cauchy-Schwarz and the inequality $2 \sqrt{\kappa} b \sqrt{\kappa} c \leq \kappa b^{2}+\kappa c^{2}$, with first $b=\left\|X^{\kappa}(s, \cdot)-X(s, \cdot)\right\|_{L^{2}}, c=$ $\|X(s, \cdot)\|_{L^{2}}$ and then $b=\left\|X^{\kappa}(s, \cdot)-X(s, \cdot)\right\|_{H^{-2}}, c=\|X(s, \cdot)\|_{L^{2}}$. We also take into account the property of $H^{-1}$ multiplier for $e^{i}, 0 \leq i \leq N$. Consequently there is a constant $\mathcal{C}(e)$ depending on $\left(e^{i}, 0 \leq i \leq N\right)$ such that

$$
g^{\kappa}(t)+2 \int_{0}^{t}\left\langle\left(X^{\kappa}-X\right)(s, \cdot), \psi\left(X^{\kappa}(s, \cdot)\right)-\psi(X(s, \cdot))\right\rangle_{L^{2}} \mathrm{~d} s
$$




$$
\begin{aligned}
& +2 \kappa \int_{0}^{t}\left\|X^{\kappa}(s, \cdot)-X(s, \cdot)\right\|_{L^{2}}^{2} \mathrm{~d} s \\
\leq & \kappa \int_{0}^{t}\left\|\left(X^{\kappa}-X\right)(s, \cdot)\right\|_{L^{2}}^{2} \mathrm{~d} s+\kappa \int_{0}^{t} \mathrm{~d} s\|X(s, \cdot)\|_{L^{2}}^{2} \\
& +\mathrm{C}(e) \int_{0}^{t} \mathrm{~d} s\left\|X^{\kappa}(s, \cdot)-X(s, \cdot)\right\|_{H^{-1}}^{2} \\
& +2 \int_{0}^{t}\left\|\left(X^{\kappa}-X\right)(s, \cdot)\right\|_{H^{-2}}\left\|\psi\left(X^{\kappa}(s, \cdot)\right)-\psi(X(s, \cdot))\right\|_{L^{2}} \\
& +2 \kappa \int_{0}^{t} \mathrm{~d} s g^{\kappa}(s)+\kappa \int_{0}^{t} \mathrm{~d} s\left\|\left(X^{\kappa}-X\right)(s, \cdot)\right\|_{H^{-2}}^{2}+\kappa \int_{0}^{t} \mathrm{~d} s\|X(s, \cdot)\|_{L^{2}}^{2}+M_{t}^{\kappa} .
\end{aligned}
$$

Since $\psi$ is Lipschitz, it follows $\left(\psi(r)-\psi\left(r_{1}\right)\right)\left(r-r_{1}\right) \geq \alpha\left(\psi(r)-\psi\left(r_{1}\right)\right)^{2}$, for any $r, r_{1} \geq 0$, for some $\alpha>0$. Consequently, the inequality $2 b c \leq b^{2} \alpha+\frac{c^{2}}{\alpha}$, with $b, c \in \mathbb{R}$ and the fact that $\|\cdot\|_{H^{-2}} \leq\|\cdot\|_{H^{-1}}$ give

$$
\begin{aligned}
& 2 \int_{0}^{t} \mathrm{~d} s\left\|\left(X^{\kappa}-X\right)(s, \cdot)\right\|_{H^{-2}}\left\|\psi\left(X^{\kappa}(s, \cdot)\right)-\psi(X(s, \cdot))\right\|_{L^{2}} \\
& \quad \leq \int_{0}^{t} \mathrm{~d} s \alpha g^{\kappa}(s, \cdot)+\int_{0}^{t} \mathrm{~d} s\left\langle\psi\left(X^{\kappa}(s, \cdot)\right)-\psi(X(s, \cdot)), X^{\kappa}(s, \cdot)-X(s, \cdot)\right\rangle_{L^{2}} .
\end{aligned}
$$

So (7.5) yields

$$
\begin{aligned}
g^{\kappa}(t) & +\int_{0}^{t}\left\langle X^{\kappa}(s, \cdot)-X(s, \cdot), \psi\left(X^{\kappa}(s, \cdot)\right)-\psi(X(s, \cdot))\right\rangle_{L^{2}} \mathrm{~d} s \\
& +\kappa \int_{0}^{t} \mathrm{~d} s\left\|X^{\kappa}(s, \cdot)-X(s, \cdot)\right\|_{L^{2}}^{2} \mathrm{~d} s \\
\leq & 2 \kappa \int_{0}^{t} \mathrm{~d} s\|X(s, \cdot)\|_{L^{2}}^{2}+M_{t}^{\kappa}+(\mathrm{C}(e)+\alpha+3 \kappa) \int_{0}^{t} g^{\kappa}(s) \mathrm{d} s .
\end{aligned}
$$

Taking the expectation we get

$$
E\left(g^{\kappa}(t)\right) \leq(\mathrm{C}(e)+\alpha+3 \kappa) \int_{0}^{t} E\left(g^{\kappa}(s)\right) \mathrm{d} s+2 \kappa \int_{0}^{t} E\left(\|X(s, \cdot)\|_{L^{2}}^{2}\right) \mathrm{d} s,
$$

for every $t \in[0, T]$. By Gronwall lemma we get

$$
E\left(g_{\kappa}(t)\right) \leq 2 \kappa E\left\{\int_{0}^{t} \mathrm{~d} s\|X(s, \cdot)\|_{L^{2}}^{2}\right\} e^{(\mathrm{C}(e)+\alpha+3 \kappa) T}, \quad \forall t \in[0, T] .
$$

Taking the supremum and letting $\kappa \rightarrow 0$, item (a) of Lemma 7.3 is now established.

We go on with item (b). Since $\psi$ is Lipschitz, (7.6) implies that, for $t \in[0, T]$,

$$
\begin{aligned}
& \int_{0}^{t} \mathrm{~d} s\left\|\psi\left(X^{\kappa}(s, \cdot)\right)-\psi(X(s, \cdot))\right\|_{L^{2}}^{2} \\
& \quad \leq \frac{1}{\alpha} \mathrm{d} s\left\langle\psi\left(X^{\kappa}(s, \cdot)\right)-\psi(X(s, \cdot)), X^{(\kappa)}(s, \cdot)-X(s, \cdot)\right\rangle_{L^{2}} \\
& \quad \leq \frac{\kappa}{2 \alpha} \int_{0}^{t} \mathrm{~d} s\|X(s, \cdot)\|_{L^{2}}^{2}+\mathrm{C}(e, \alpha) \int_{0}^{t} g_{\kappa}(s) \mathrm{d} s+M_{t}^{\kappa},
\end{aligned}
$$


where $\mathrm{C}(e, \alpha)$ is a constant depending on $e^{i}, 0 \leq i \leq N$ and $\alpha$. Taking the expectation for $t=T$, we get

$$
E\left(\int_{0}^{t} \mathrm{~d} s\left\|\psi\left(X^{\kappa}(s, \cdot)\right)-\psi(X(s, \cdot))\right\|_{L^{2}}^{2}\right) \leq \frac{\kappa}{2 \alpha} E\left(\int_{0}^{t} \mathrm{~d} s\|X(s, \cdot)\|_{L^{2}}^{2}\right)+\mathrm{C}(e, \alpha) \int_{0}^{T} E\left(g_{\kappa}(s)\right) \mathrm{d} s .
$$

Taking $\kappa \rightarrow 0$, (B.2) and (7.7) provide the conclusion of item (b) of Lemma 7.3.

(c) Coming back to (7.6), and $t=T$, we have

$$
\kappa \int_{0}^{t} \mathrm{~d} s\left\|X^{\kappa}(s, \cdot)-X(s, \cdot)\right\|_{L^{2}}^{2} \leq 2 \kappa \int_{0}^{t} \mathrm{~d} s\|X(s, \cdot)\|_{L^{2}}^{2}+M_{T}^{\kappa}+(\mathrm{C}(e)+\alpha+3 \kappa) \int_{0}^{t} \mathrm{~d} s g^{\kappa}(s) .
$$

Taking the expectation we have

$$
\kappa E\left(\int_{0}^{t} \mathrm{~d} s\left\|X^{\kappa}(s, \cdot)-X(s, \cdot)\right\|_{L^{2}}^{2}\right) \leq 2 \kappa E\left(\int_{0}^{t} \mathrm{~d} s\|X(s, \cdot)\|_{L^{2}}^{2}\right)+(\mathrm{C}(e)+\alpha+3 \kappa) E\left(\int_{0}^{T} g^{\kappa}(s) \mathrm{d} s\right) .
$$

Using item (a) and the fact that $E\left(\int_{[0, T] \times \mathbb{R}} X^{2}(s, \xi) \mathrm{d} s \mathrm{~d} \xi\right)<\infty$, the result follows. Lemma 7.3 is finally completely established.

We need now another intermediate lemma concerning the paths of a solution to (1.1).

Lemma 7.5. For almost all $\omega \in \Omega$, almost all $t \in[0, T]$,

(1) $\xi \mapsto \psi(X(t, \xi, \omega)) \in H^{1}(\mathbb{R})$,

(2) $\xi \mapsto \Phi(X(t, \xi, \omega))$ is continuous.

Proof. Item (1) is established in [8], see Definition 3.2 and Theorem 3.4. (1) implies that $\xi \mapsto \psi(X(t, \xi, \omega))$ is continuous. See also Remark B.2 1. By the same arguments as in Proposition 4.22 in [6], we can deduce item (2).

(3) We go on with the proof of Theorem 7.1. We keep in mind (i), (ii), (iii), (iv) at the beginning of item (1) of the proof. Since $\Phi$ is bounded, for $P$-almost all $\omega$, using Burkholder-Davis-Gundy inequality one obtains

$$
E^{Q^{\kappa}(\cdot, \omega)}\left(Y_{t}-Y_{s}\right)^{4} \leq \operatorname{const}(t-s)^{2},
$$

where const does not depend on $\omega$. On the other hand, for all $Q^{\kappa}(\cdot, \omega), Y_{0}$ is distributed according to $x_{0}$.

At this point, we need a version of Kolmogorov-Centsov theorem for the stable convergence. Let $\tilde{\Omega}_{0}=\tilde{\Omega}_{1} \times \Omega$ as at the beginning of the proof of Theorem 7.1. We recall that $\tilde{\Omega}_{1}=C([0, T]) \times \mathbb{R}, Y\left(\omega_{1}, \omega\right)=\omega_{1}, \mathcal{H}$ is the Borel $\sigma$-field on $\tilde{\Omega}_{1}$.

Lemma 7.6. Let be a sequence $Q^{\kappa}(\cdot, \omega)$ of random kernel on $\mathcal{H} \times \Omega$. Let us denote by $\mathbf{Q}^{\kappa}$ the sequence of marginal laws of the probabilities on $\left(\tilde{\Omega}_{0}, \mathcal{H} \otimes \mathcal{F}\right)$ given by $Q^{\kappa}(\cdot, \omega) P(\mathrm{~d} \omega)$. Suppose the following.

- The sequences of marginal laws of the probabilities $\mathbf{Q}^{\kappa}$ at zero are tight.

- There are $\alpha, \beta>0$ such that

$$
E^{Q^{\kappa}(\cdot, \omega)}\left|Y_{t}-Y_{S}\right|^{\alpha} \leq C(\omega)(t-s)^{1+\beta}, \quad 0 \leq s \leq T,
$$

for some positive $P$-integrable random constant $C$.

Then there is a random kernel $Q^{\infty}$ on $\mathcal{H} \times \Omega$ and a subsequence $\left(\kappa_{n}\right)$ such that for every bounded continuous functional $G: \tilde{\Omega}_{1} \rightarrow \mathbb{R}$, for every bounded $\mathcal{F}$-measurable r.v. $F: \Omega \rightarrow \mathbb{R}$, we have

$$
\begin{aligned}
& \int_{\Omega} F(\omega) \mathrm{d} P(\omega) \int_{\tilde{\Omega}_{1}} G\left(Y\left(\omega_{1}\right)\right) Q^{\kappa_{n}}\left(\mathrm{~d} \omega_{1}, \omega\right) \\
& \quad \underset{n \rightarrow \infty}{\rightarrow} \int_{\Omega} F(\omega) \mathrm{d} P(\omega) \int_{\tilde{\Omega}_{1}} G\left(Y\left(\omega_{1}\right)\right) Q^{\infty}\left(\mathrm{d} \omega_{1}, \omega\right) .
\end{aligned}
$$


Proof. Taking the expectation with respect to $P$ we obtain

$$
E^{\mathbf{Q}^{\kappa}}\left(Y_{t}-Y_{s}\right)^{\alpha} \leq C_{0}(t-s)^{1+\beta}, \quad 0 \leq s \leq T,
$$

where $C_{0}$ is the expectation of $C$. First, by usual arguments as Chebyshev inequality, one can show the following:

$$
\begin{aligned}
& \lim _{\lambda \rightarrow \infty} \sup _{\kappa} \mathbf{Q}^{\kappa}\left\{\left(\omega_{1}, \omega\right)||\left(W^{1}, \ldots, W^{N}\right)(\omega)(0)|>\lambda ;| \omega_{1}(0) \mid>\lambda\right\}=0, \\
& \lim _{\delta \rightarrow 0} \sup _{\kappa} \mathbf{Q}^{\kappa}\left\{\left(\omega_{1}, \omega\right) \mid m\left(\left(W^{1}, \ldots, W^{N}, \omega_{1}\right) ; \delta\right)>\varepsilon\right\}=0, \quad \forall \varepsilon>0,
\end{aligned}
$$

where $m$ denotes the modulus of continuity. By Theorem 4.10 of [18], the sequences of probabilities $\mathbf{Q}^{\kappa}, \kappa>0$, on $\tilde{\Omega}_{1} \times \Omega$ are tight. Let $\mathbf{Q}^{\kappa_{n}}$ be a sequence converging weakly to a probability $\mathbf{Q}^{\infty}$ on $\mathcal{H} \otimes \mathcal{F}$. Since $\mathcal{F}$ is separable and $C([0, T])^{N}$, which is space value of process $W$, is a Polish space equipped with its Borel $\sigma$ algebra, according to [23], it is possible to desintegrate $\mathbf{Q}^{\infty}$, i.e. there is random kernel $Q^{\infty}(\cdot, \omega)$ such that for every bounded continuous functional $G: \tilde{\Omega}_{1} \rightarrow \mathbb{R}$, for every bounded continuous $\tilde{F}: C([0, T])^{N} \rightarrow \mathbb{R}$ such that (7.9) holds for every $F=\tilde{F}(W)$, where $W=\left(W^{1}, \ldots, W^{N}\right)$. Since continuous bounded functionals $\tilde{F}$ are dense in $L^{2}\left(C([0, T])^{N}\right)$ equipped with Wiener measure, (7.9) holds also for any $F$ bounded $\mathcal{F}$-measurable r.v. with $\mathbf{Q}^{\infty}\left(\mathrm{d} \omega_{1}, \mathrm{~d} \omega\right)=Q^{\infty}\left(\mathrm{d} \omega_{1}, \omega\right) P(\mathrm{~d} \omega)$.

By (7.8), we apply Lemma 7.6 with $\alpha=2, \beta=1$ and we consider the corresponding $Q^{\kappa_{n}}(\cdot, \omega)$ and the limit random kernel $Q(\cdot, \omega):=Q^{\infty}(\cdot, \omega)$. We define also the probability $\mathbf{Q}:=\mathbf{Q}^{\infty}$ on $\tilde{\Omega}_{0}=\tilde{\Omega}_{1} \times \Omega$ according to the conventions introduced before Definition 2.3. In the sequel we denote again by $\mathrm{d} \mathbf{Q}^{\kappa}\left(\omega_{1}, \omega\right):=\mathrm{d} P(\omega) Q^{\kappa}\left(\mathrm{d} \omega_{1}, \omega\right)$ and also $Q^{\omega, \kappa}:=Q^{\kappa}(\cdot, \omega), Q^{\omega}:=Q(\cdot, \omega)$.

From Lemma 7.6 derives the following.

Corollary 7.7. For any bounded random element $F: \tilde{\Omega}_{1} \times \Omega \rightarrow \mathbb{R}$ such that for almost all $\omega \in \Omega, F(\cdot, \omega) \in$ $C\left(\tilde{\Omega}_{1}\right)$. Then $\int_{\Omega} \mathrm{d} P(\omega) \int_{\tilde{\Omega}_{1}}\left(\mathrm{~d} Q^{\omega, \kappa_{n}}\left(\omega_{1}\right)-\mathrm{d} Q^{\omega}\left(\omega_{1}\right)\right) F(Y, \omega)$ converges to zero.

Proof. See Appendix A.

We need here a technical lemma.

Lemma 7.8. Let $t \in[0, T], p \in \mathbb{R}$.

1. There is $C(p)>0$ such that

$$
E^{\mathbf{Q}^{\kappa}}\left(\mathcal{E}_{t}\left(\int_{0} \mu\left(\mathrm{d} s, Y_{s}\right)\right)^{p}\right) \leq C(p), \quad \forall \kappa>0 .
$$

2. For almost all $\omega \in \Omega$, and every $p \in \mathbb{R}$ there is a random constant $C(p, \omega)$ such that the random variables

$$
E^{Q^{\omega, \kappa}}\left(\mathcal{E}_{t}\left(\int_{0}^{\cdot} \mu\left(\mathrm{d} s, Y_{s}\right)\right)^{p}\right) \leq C(p, \omega), \quad \forall \kappa>0 .
$$

Proof. Without restriction of generality we can of course suppose $e^{0}=0$.

1. We can write

$$
\begin{aligned}
\mathcal{E}_{t}\left(\int_{0} \mu\left(\mathrm{d} s, Y_{s}\right)\right)^{p} & =\mathcal{E}_{t}\left(p \int_{0}^{\cdot} \mu\left(\mathrm{d} s, Y_{s}\right)\right) \exp \left(\frac{p^{2}-p}{2} \sum_{i=1}^{N}\left(\int_{0}^{t} e^{i}\left(Y_{s}\right)^{2} \mathrm{~d} s\right)\right) \\
& \leq \mathcal{E}_{t}\left(p \int_{0} \mu\left(\mathrm{d} s, Y_{s}\right)\right) \exp \left(T \frac{p^{2}-p}{2} \sum_{i=1}^{N}\left\|e^{i}\right\|_{\infty}^{2}\right) .
\end{aligned}
$$

Since $p \int_{0}^{t} \mu\left(\mathrm{d} s, Y_{s}\right)$ is a $\left(\mathcal{G}_{t}\right)-\mathbf{Q}^{\kappa}$-martingale, the result follows. 
2. Let $\omega \in \Omega$ excepted on a $P$-null set. The integrand of the expectation in (7.10) equals $\exp \left(J_{1}(n)+J_{2}(n)\right)$, where

$$
J_{1}(n):=p \sum_{i=1}^{N}\left(W_{t}^{i} e^{i}\left(Y_{t}\right)-\frac{1}{2} \int_{0}^{t} e^{i}\left(Y_{S}\right)^{2} \mathrm{~d} s-\frac{1}{2} \int_{0}^{t} W_{s}^{i}\left(e^{i}\right)^{\prime \prime}\left(Y_{S}\right) \Phi^{2}\left(X\left(s, Y_{s}, \omega\right)\right) \mathrm{d} s\right)
$$

and $J_{2}(n)=-p \sum_{i=1}^{N} \int_{0}^{t} W_{s}^{i}\left(e^{i}\right)^{\prime}\left(Y_{s}\right) \mathrm{d} Y_{s}$. For each $\omega, \exp \left(J_{1}(n)\right)$ is bounded, so it remains to prove the existence of a random constant $C(p, \omega)$ such that for every $0 \leq i \leq N$

$$
E^{Q^{\omega, \kappa}}\left(\exp \left(-p \int_{0}^{t} W_{s}^{i}\left(e^{i}\right)^{\prime}\left(Y_{s}\right) \mathrm{d} Y_{s}\right)\right) \leq C(p, \omega)
$$

Since $-p \int_{0}^{t} W_{s}^{i}\left(e^{i}\right)^{\prime}\left(Y_{s}\right) \mathrm{d} Y_{s}$ is a $Q^{\omega, \kappa}$-martingale,

$$
\mathcal{E}_{t}^{\kappa}:=\exp \left(-p \int_{0}^{t} W_{s}^{i}\left(e^{i}\right)^{\prime}\left(Y_{s}\right) \mathrm{d} Y_{s}-\frac{p^{2}}{2} \int_{0}^{t}\left(W^{i}\right)_{s}^{2}\left(e^{i}\right)^{\prime 2}\left(Y_{s}\right) \Phi_{\kappa}^{2}\left(X^{\kappa}\left(s, Y_{s}, \omega\right)\right) \mathrm{d} s\right)
$$

is an (exponential) martingale, with respect to $Q^{\omega, \kappa}$. Consequently the left-hand side of (7.11) is bounded by

$$
\begin{gathered}
E^{Q^{\omega, \kappa}}\left(\mathcal{E}_{t}^{\kappa} \exp \left(\frac{p^{2}}{2} \int_{0}^{t}\left(W^{i}\right)_{s}^{2}\left(\left(e^{i}\right)^{\prime}\right)^{2}\left(Y_{s}\right) \Phi_{\kappa}^{2}\left(X^{\kappa}\left(s, Y_{s}, \omega\right)\right) \mathrm{d} s\right)\right) \\
\leq C(p, \cdot):=\exp \left(\frac{p^{2}}{2}\left\|\left(e^{i}\right)^{\prime}\right\|_{\infty}^{2}\left(\|\Phi\|_{\infty}^{2}+1\right) \int_{0}^{T}\left(W_{s}^{i}\right)^{2} \mathrm{~d} s\right) .
\end{gathered}
$$

This concludes the proof of Lemma 7.8.

Lemma 7.9. We fix $\omega \in \Omega$ excepted on some P-null set. Let $\varphi:[0, T] \times \mathbb{R} \rightarrow \mathbb{R}$ continuous with compact support. The random variables

$$
E^{Q^{\omega, \kappa}}\left(\int_{0}^{T}\left|\Phi_{\kappa}\left(X^{\kappa}\left(r, Y_{r}, \omega\right)\right)-\Phi\left(X\left(r, Y_{r}, \omega\right)\right)\right| \varphi\left(r, Y_{r}\right) \mathrm{d} r\right)
$$

converge to zero a.s. and in $L^{p}(\Omega, P)$ for every $p \geq 1$, when $\kappa \rightarrow 0$.

Proof. Let $\omega \in \Omega$. Since $\varphi$ has compact support, by Cauchy-Schwarz with respect to the measure $\varphi(r, Y(r)) \mathrm{d} r$ on $[0, T]$, it is enough to prove that

$$
E^{Q^{\omega, \kappa}}\left(\int_{0}^{T}\left(\Phi_{\kappa}\left(X^{\kappa}\left(r, Y_{r}, \omega\right)\right)-\Phi\left(X\left(r, Y_{r}, \omega\right)\right)\right)^{2} \varphi\left(r, Y_{r}\right) \mathrm{d} r\right)
$$

converges to zero. Since $\Phi$ is bounded it is enough to prove the convergence to zero for almost all $\omega \in \Omega$. In order not to overcharge the notation, in this proof we will omit the argument of $\omega$ of $Y$. By Fubini's theorem the left-hand side of (7.12) equals

$$
\int_{0}^{T} \mathrm{~d} r E^{Q^{\omega, \kappa}}\left(\left(\Phi_{\kappa}\left(X^{\kappa}\left(r, Y_{r}, \omega\right)\right)-\Phi\left(X\left(r, Y_{r}, \omega\right)\right)\right)^{2} \varphi\left(r, Y_{r}\right)\right) .
$$

Using also Lebesgue dominated convergence theorem, given a sequence $\left(\kappa_{n}\right)$, when $n \rightarrow \infty$, it is enough to find a subsequence $\left(\kappa_{n_{\ell}}\right)$ such that for all $r \in[0, T]$ outside a possible Lebesgue null set

$$
E^{Q^{\omega, \kappa_{\ell}}}\left\{\left(\Phi_{\kappa_{n_{\ell}}}\left(X^{\kappa_{n_{\ell}}}\left(r, Y_{r}, \omega\right)\right)-\Phi\left(X\left(r, Y_{r}, \omega\right)\right)\right)^{2} \varphi\left(r, Y_{r}\right)\right\}_{\ell \rightarrow \infty}^{\rightarrow} 0 .
$$

We set $Z_{r}\left(\omega_{1}, \omega\right)=\mathcal{E}_{r}\left(\int_{0}^{\cdot} \mu(\omega)\left(\mathrm{d} s, Y_{s}\left(\omega_{1}\right)\right)\right)$. We will substitute from now on $\left(n_{\ell}\right)$ with $n$. 
Taking into account Lemma 7.8 and Cauchy-Schwarz with respect to the finite measure $Z_{r}\left(\omega_{1}, \omega\right) Q^{\omega, \kappa_{n}}\left(\mathrm{~d} \omega_{1}\right)$, it is enough to prove that for $r$ a.e.

$$
E^{Q^{\omega, \kappa_{n}}}\left\{\left(\Phi_{\kappa_{n}}\left(X^{\kappa_{n}}\left(r, Y_{r}, \omega\right)\right)-\Phi\left(X\left(r, Y_{r}, \omega\right)\right)\right)^{2} \varphi\left(r, Y_{r}\right) Z_{r}(\cdot, \omega)\right\}
$$

converges to zero when $n$ goes to infinity.

Since $X^{\kappa}$ constitutes the family of $\mu$-marginal weighted laws of $Y$ under $Q^{\omega, \kappa}$, previous expression gives

$$
\begin{aligned}
& \int_{\mathbb{R}}|\varphi|(r, y)\left(\Phi_{\kappa_{n}}\left(X^{\kappa_{n}}(r, y, \omega)\right)-\Phi(X(r, y, \omega))\right)^{2} X^{\kappa_{n}}(r, y, \omega) \mathrm{d} y \\
& \quad \leq I_{11}\left(\kappa_{n}, r\right)+I_{12}\left(\kappa_{n}, r\right)+I_{13}\left(\kappa_{n}, r\right)+I_{14}\left(\kappa_{n}, r\right),
\end{aligned}
$$

where we have developed the square in the first line of (7.13) using the definition of $\psi$ and $\Phi_{\kappa}$. Indeed we get

$$
\begin{aligned}
& I_{11}(\kappa, r)=\int_{\mathbb{R}} \mathrm{d} y|\varphi|(r, y)|| \psi\left(X^{\kappa}(r, y, \omega)\right)-\psi(X(r, y, \omega)) \mid, \\
& I_{12}(\kappa, r)=\int_{\mathbb{R}} \mathrm{d} y|\varphi|(r, y)\left|\Phi^{2}\left(X^{\kappa}(r, y, \omega)\right)\right|\left(X-X^{\kappa}\right)(r, y, \omega) \mid, \\
& I_{13}(\kappa, r)=\int_{\mathbb{R}} \mathrm{d} y|\varphi(r, y)| \kappa\left|X^{\kappa_{n}}-X\right|(r, y, \omega), \\
& I_{14}(\kappa, r)=\int_{0}^{t} \mathrm{~d} r \int_{\mathbb{R}} \mathrm{d} y \kappa|X(r, y, \omega)||\varphi(r, y)| .
\end{aligned}
$$

We denote $I_{1 j}(\kappa):=\int_{0}^{T} I_{1 j}(\kappa, r) \mathrm{d} r, j=1,2,3,4$. It is of course enough to prove that, up to a subsequence $I_{1 j}\left(\kappa_{n}\right) \rightarrow$ $0, j=1,2,3,4$, where $n \rightarrow \infty$. By Cauchy-Schwarz, $I_{11}^{2}(\kappa)$ is bounded by

$$
\|\varphi\|_{L^{2}([0, T] \times \mathbb{R})}^{2} \int_{0}^{t} \mathrm{~d} r \int_{\mathbb{R}}\left(\psi\left(X^{\kappa}(r, y, \omega)\right)-\psi(X(r, y, \omega))\right)^{2} \mathrm{~d} y .
$$

This converges to zero according to Remark 7.4(2), after extracting a subsequence $\left(\kappa_{n}\right)$ (not depending on $\omega$ ). The square of the expectation of $I_{12}(\kappa)$ is bounded by

$$
\|\varphi\|_{L^{2}([0, T] \times \mathbb{R})}^{2} \int_{[0, T] \times \mathbb{R}} \mathrm{d} r \mathrm{~d} y \Phi^{4}(X(r, y, \omega))\left|X^{\kappa}-X\right|^{2}(r, y, \omega) .
$$

The expectation of previous expression is indeed uniformly bounded in $\kappa$ because of (7.6) and (7.7). So the family of r.v. $\Phi^{2}\left(X^{\kappa_{n}}(r, y, \omega)\right)\left|\left(X-X^{\kappa}\right)(r, y, \omega)\right|$ is uniformly integrable with respect to the finite measure $\mathrm{d} P(\omega)|\varphi|(t, y) \mathrm{d} t \mathrm{~d} y$. Consequently $I_{12}(\kappa)$ goes to zero because of (7.3) in Remark 7.4(4).

$I_{13}^{2}(\kappa)$ is bounded by $\kappa\|\varphi\|_{L^{2}([0, T] \times \mathbb{R})}^{2} \kappa \int_{[0, T] \times \mathbb{R}} \mathrm{d} r \mathrm{~d} y\left|X^{\kappa}-X\right|^{2}(r, y, \omega)$. After extracting a subsequence $\kappa_{n}$, previous expression converges to zero because of Lemma 7.3(c). Finally $I_{14}(\kappa) \rightarrow_{n \rightarrow \infty} 0$ by Cauchy-Schwarz and the fact that $\int_{[0, T] \times \mathbb{R}} \mathrm{d} r \mathrm{~d} y X^{2}(r, y, \omega)<\infty \mathrm{d} P$-a.s. This establishes the proof of Lemma 7.9.

Let $\left(\kappa_{n}\right)$ be the sequence introduced by the statement of Lemma 7.6. Previous Corollary 7.7 and Lemma 7.9 have the following consequences. Let $Q\left(\mathrm{~d} \omega_{1}, \omega\right)$ be the random kernel introduced in Lemma 7.5 and the related probability $\mathbf{Q}\left(\mathrm{d} \omega_{1}, \mathrm{~d} \omega\right)=\mathrm{d} P(\omega) Q\left(\mathrm{~d} \omega_{1}, \omega\right)$.

Corollary 7.10. Let $R: \Omega \rightarrow \mathbb{R}$ be a bounded measurable r.v. Let $\varphi:[0, T] \times \mathbb{R} \rightarrow \mathbb{R}$ be a function with compact support. The sequence

$$
\int_{\Omega} R(\omega) \mathrm{d} P(\omega) \int_{\tilde{\Omega}_{1}} \mathrm{~d} Q^{\omega, \kappa_{n}}\left(\omega_{1}\right) \int_{0}^{T} \varphi\left(r, Y_{r}\right) \Phi_{\kappa_{n}}^{2}\left(X^{\kappa_{n}}\left(r, Y_{r}, \omega\right)\right) \mathrm{d} r
$$


converges, when $n \rightarrow \infty$, to

$$
\int_{\Omega} R(\omega) \mathrm{d} P(\omega) \int_{\tilde{\Omega}_{1}} \mathrm{~d} Q\left(\omega_{1}, \omega\right) \int_{0}^{T} \varphi\left(r, Y_{r}\right) \Phi^{2}\left(X\left(r, Y_{r}, \omega\right)\right) \mathrm{d} r .
$$

Proof. We split the difference between (7.14) and (7.15) which gives $I_{1}(n)+I_{2}(n)$ where

$$
I_{1}(n)=\int_{\Omega} R(\omega) \mathrm{d} P(\omega) \int_{\tilde{\Omega}_{1}} \mathrm{~d} Q^{\omega, \kappa_{n}}\left(\omega_{1}\right) \int_{0}^{T} \varphi\left(r, Y_{r}\right)\left(\Phi_{\kappa_{n}}^{2}\left(X^{\kappa_{n}}\left(r, Y_{r}, \omega\right)\right) \mathrm{d} r-\Phi^{2}\left(X\left(r, Y_{r}, \omega\right)\right) \mathrm{d} r\right),
$$

and

$$
I_{2}(n)=\int_{\Omega} R(\omega) \mathrm{d} P(\omega) \int_{\tilde{\Omega}_{1}}\left(Q^{\omega, \kappa_{n}}\left(\mathrm{~d} \omega_{1}\right)-Q\left(\mathrm{~d} \omega_{1}, \omega\right)\right)\left(\int_{0}^{T} \varphi\left(r, Y_{r}\right) \Phi^{2}\left(X\left(r, Y_{r}, \omega\right)\right) \mathrm{d} r\right) .
$$

We have

$$
\begin{aligned}
\left|I_{1}(n)\right| \leq & 2\|\Phi\|_{\infty}\|R\|_{\infty} \\
& \times \int_{\Omega} \mathrm{d} P(\omega) \int_{\tilde{\Omega}_{1}} \mathrm{~d} Q^{\omega, \kappa_{n}}\left(\omega_{1}\right)\left(\int_{0}^{T}\left|\varphi\left(r, Y_{r}\right) \| \Phi_{\kappa_{n}}\left(X^{\kappa_{n}}\left(r, Y_{r}, \omega\right)\right) \mathrm{d} r-\Phi\left(X\left(r, Y_{r}, \omega\right)\right)\right| \mathrm{d} r\right) .
\end{aligned}
$$

$I_{1}(n)$ converges to zero by Lemma 7.9. Concerning $I_{2}(n)$, by Fubini's theorem, we first observe that

$$
I_{2}(n)=\int_{0}^{T} \mathrm{~d} r \int_{\Omega} \mathrm{d} P(\omega)\left(\int_{\tilde{\Omega}_{1}}\left(Q^{\omega, \kappa_{n}}\left(\mathrm{~d} \omega_{1}\right)-Q\left(\mathrm{~d} \omega_{1}, \omega\right)\right) \varphi\left(r, Y_{r}\right) \Phi^{2}\left(X\left(r, Y_{r}, \omega\right)\right) R(\omega)\right) .
$$

We apply now Corollary 7.7, setting for fixed $r, F\left(\omega_{1}, \omega\right)=R(\omega) \varphi\left(r, \omega_{1}(r)\right) \Phi^{2}\left(X\left(r, \omega_{1}(r), \omega\right)\right)$ and the result follows.

(4) We go on with the proof of Theorem 7.1.

We want now to prove that $Y(\cdot, \omega)$ is a (weak) solution of

$$
Y_{t}=Y_{0}+\int_{0}^{t} \Phi\left(X\left(s, Y_{s}, \cdot\right)\right) \mathrm{d} \beta_{s}^{\omega}
$$

for some Brownian motion $\beta^{\omega}$. This is related to item (1) of Definition 2.10 with $\gamma(t, \xi, \omega)=\Phi(X(t, \xi, \omega))$. According to Remark 2.11(c), for this it is enough to show that for $\mathrm{d} P$-a.s. $\omega Y(\cdot, \omega)$ is a solution of the following (local) martingale problem. For every $f \in C^{1,2}([0, T] \times \mathbb{R})$ with compact support, the process

$$
Z_{t}^{f}:=f\left(t, Y_{t}\right)-f\left(0, Y_{0}\right)-\frac{1}{2} \int_{0}^{t} \partial_{x x}^{2} f\left(r, Y_{r}\right) \Phi^{2}(X(r, Y, \omega)) \mathrm{d} r-\int_{0}^{t} \partial_{r} f\left(r, Y_{r}\right) d r,
$$

is a (local) martingale under $Q^{\omega}$.

This will be a consequence of the lemma below.

Lemma 7.11. Let $F$ be a bounded $\mathcal{F}_{s}$-measurable, let $\mathcal{A}: C([0, s]) \rightarrow \mathbb{R}$ bounded continuous functional. Let $G=$ $\mathcal{A}\left(Y_{r}, r \leq s\right)$. Then, for $0 \leq s \leq t \leq T$ we have

$$
E\left(F E^{Q^{\omega}}\left(G Z_{t}^{f}\right)\right)=E\left(F E^{Q^{\omega}}\left(G Z_{s}^{f}\right)\right)
$$

Proof. We set

$$
Z_{t}^{\kappa, f}=f\left(t, Y_{t}\right)-f\left(0, Y_{0}\right)-\frac{1}{2} \int_{0}^{t} \partial_{x x}^{2} f\left(r, Y_{r}\right) \Phi_{\kappa}^{2}\left(X^{\kappa}(r, Y, \omega)\right) \mathrm{d} r-\int_{0}^{t} \partial_{r} f\left(r, Y_{r}\right) \mathrm{d} r .
$$


Let $\left(\kappa_{n}\right)$ be the sequence introduced by Lemma 7.6. The difference of the right and left-hand side of (7.16) is the sum $\left(I_{1}+I_{2}+I_{3}\right)\left(\kappa_{n}\right)$ where

$$
\begin{aligned}
& I_{1}(\kappa)=E\left(F\left(E^{Q^{\omega}}\left(G Z_{t}^{f}\right)-E^{Q^{\omega, \kappa}}\left(G Z_{t}^{\kappa, f}\right)\right)\right), \\
& I_{2}(\kappa)=E\left(F E^{Q^{\omega, \kappa}}\left(G\left(Z_{t}^{\kappa, f}-Z_{s}^{\kappa, f}\right)\right)\right), \\
& I_{3}(\kappa)=E\left(F\left(E^{Q^{\omega, \kappa}}\left(G Z_{s}^{\kappa, f}\right)-E^{Q^{\omega}}\left(G Z_{s}^{f}\right)\right)\right) .
\end{aligned}
$$

$I_{1}\left(\kappa_{n}\right)+I_{3}\left(\kappa_{n}\right)$ converges to zero by Lemma 7.6, Corollary 7.10 and Lemma 7.9. $I_{2}\left(\kappa_{n}\right)=0$ since $Z^{\kappa, f}$ is a $Q^{\kappa, \omega_{-}}$ martingale.

(5) After previous intermediary steps we need to show that $Y$ defined in (7.1) is a weak-strong solution of $\operatorname{DSDE}\left(\gamma, x_{0}\right)$ with $\gamma(s, \xi, \omega)=\Phi(X(s, \xi, \omega))$ and $X$ is a solution of $(1.1)$. We recall that the kernel $Q(\cdot, \omega)$ has been introduced through Lemma 7.6 on $\left(\tilde{\Omega}_{1} \times \Omega, \mathcal{H} \otimes \mathcal{F}\right)$. So, according to step (5), under $Q^{\omega}:=Q(\cdot, \omega), Y$ is a martingale with $[Y]_{t}=\int_{0}^{t} \Phi^{2}\left(X\left(s, Y_{s}, \omega\right)\right) \mathrm{d} s$. To conclude the proof of item (1) in Definition 2.10, it remains to construct the suitable required process $B$. For this, we need to enlarge the probability space $\tilde{\Omega}_{1}$ as follows. We set $\Omega_{1}=\tilde{\Omega}_{1} \times C([0, T] ; \mathbb{R})$; the second component allows to define a Brownian motion. By an abuse of notation, we set again $Y_{t}\left(\omega_{1}, \omega\right)=\omega_{1}^{0}(t)$, this time with $\omega_{1}=\left(\omega_{1}^{0}, \omega_{1}^{1}\right)$. In spite of adding the component $\omega_{1}^{1}$, in step (5) we have already shown $Q^{\omega}:=Q(\cdot, \omega)$, is by construction the law of $Y(\cdot, \omega)$. We need to construct a process $B$ on $\Omega \times \Omega_{1}$, such that for almost all $\omega, B(\cdot, \omega)$ is a $Q^{\omega}$-Brownian motion and (2.6) holds for $\gamma(t, \cdot, \omega)=\Phi(X(t, \cdot, \omega))$.

On $\Omega_{1}$ we set $\beta_{t}\left(\omega_{1}\right)=\omega_{1}^{1}(t)$. We equip $C([0, T] ; \mathbb{R})$ in $\Omega_{1}$ with the Wiener measure $\mathcal{W}$ so that $\beta$ is a standard Brownian motion on $\Omega_{1}$. $\beta$ can also be considered to be a Brownian motion on $\Omega_{0}=\Omega_{1} \times \Omega$ which is $Q^{\omega}$-independent of $Y$ for $P$-almost all $\omega \in \Omega$. Of course $\beta$ is also independent of $Y$ on the probability space $\left(\Omega_{1} \times \Omega, \mathcal{B}\left(\Omega_{1}\right) \times \mathcal{F}, \mathrm{d} \mathbf{Q}\left(\omega_{1}, \omega\right):=Q^{\omega}\left(\mathrm{d} \omega_{1}\right) \mathrm{d} P(\omega)\right) . \beta$ is also independent of $\left(\mathcal{F}_{t}\right)$.

We set now

$$
B_{t}(\cdot, \omega)=\int_{0}^{t} \mathrm{~d} Y_{s}(\cdot, \omega) 1_{\{\gamma(s, \xi, \omega) \neq 0\}} \frac{1}{\gamma(s, \xi, \omega)}+\int_{0}^{t} 1_{\{\gamma(s, \xi, \omega)=0\}} \mathrm{d} \beta_{s} .
$$

Now for $Q^{\omega}$-a.s. the quadratic variation of the $Q^{\omega}$-martingale $B(\cdot, \omega)$ is $t$, so that, by Lévy characterization theorem, $B(\cdot, \omega)$ is a Brownian motion under $Q^{\omega}$.

It remains to show items (2) and (3) of the definition of weak-strong solution. Let $\left(\mathcal{Y}_{t}\right)$ be the canonical filtration of the process $Y(\cdot, \omega)$. Item (3) follows because of item (1) and because $\gamma(t, \cdot, \omega)=\Phi(X(t, \cdot, \omega))$ is progressively measurable. Concerning item (2) we see that under $\mathbf{Q}$ defined by $P$ and the kernel $Q(\cdot, \omega), W^{1}, \ldots, W^{N}$ are $\mathbf{Q}-$ martingales with $\left(\mathcal{G}_{t}\right)$ as defined in Definition 2.10. Indeed let $F$ be a bounded $\mathcal{F}_{s}$-measurable random variable and $G$ be a bounded $\mathcal{Y}_{s}$-measurable r.v. Let $1 \leq i \leq N$. By item $(3) E^{Q^{\omega}}(G)$ is $\mathcal{F}_{s}$-measurable, so

$$
E^{\mathbf{Q}}\left(\left(W_{t}^{i}-W_{s}^{i}\right) F G\right)=E\left(\left(W_{t}^{i}-W_{s}^{i}\right) F E^{Q^{\omega}}(G)\right)=0,
$$

since $W^{i}$ is an $\mathcal{F}_{s}$-martingale.

(6) The final step consists in proving that $X$ is the family of $\mu$-marginal weighted laws of $Y$. We need to show that for almost all $\omega$, for every $t \in[0, T], \varphi \in \mathcal{S}(\mathbb{R})$, that

$$
\int_{\mathbb{R}} \mathrm{d} \xi \varphi(\xi) X(t, \xi, \omega)=E^{Q^{\omega}}\left(\varphi\left(Y_{t}\right) \mathcal{E}_{t}\left(\int_{0}^{\cdot} \mu\left(\mathrm{d} s, Y_{s}\right)\right)\right) .
$$

Since both sides of previous equality are $\mathcal{F}_{t}$-measurable, given a bounded $\mathcal{F}_{t}$-measurable random variable $R$ it will be enough to show that

$$
\int_{\Omega} \mathrm{d} P(\omega) R(\omega) \int_{\mathbb{R}} \mathrm{d} \xi \varphi(\xi) X(t, \xi, \omega)=\int_{\Omega} \mathrm{d} P(\omega) R(\omega) E^{Q^{\omega}}\left(\varphi\left(Y_{t}\right) \mathcal{E}_{t}\left(\int_{0} \mu\left(\mathrm{d} s, Y_{s}\right)\right)\right) .
$$

Let $\omega \in \Omega$ outside some $P$-null set. 
By step (1) of the proof of this Theorem 7.1, we know that $X^{\kappa}$ fulfills, for almost all $\omega$,

$$
\int_{\mathbb{R}} \mathrm{d} \xi X^{\kappa}(t, \xi) \varphi(\xi)=E^{Q^{\kappa}(\cdot, \omega)}\left(\varphi\left(Y_{t}\right) \mathcal{E}_{t}\left(\int_{0}^{\cdot} \mu\left(\mathrm{d} s, Y_{s}\right)\right)\right),
$$

for all $\varphi \in \mathcal{S}(\mathbb{R})$. Consequently if $\left(\kappa_{n}\right)$ is the sequence obtained via Lemma 7.5, we have

$$
\int_{\Omega} \mathrm{d} P(\omega) R(\omega) \int_{\mathbb{R}} \mathrm{d} \xi X^{\kappa_{n}}(t, \xi) \varphi(\xi)=\int_{\Omega} \mathrm{d} P(\omega) R(\omega) E^{Q^{\omega, \kappa_{n}}}\left(\varphi\left(Y_{t}\right) \mathcal{E}_{t}\left(\int_{0} \mu(\mathrm{d} s, Y)\right)\right),
$$

for every $\varphi \in \mathcal{S}(\mathbb{R})$.

Since $t \mapsto X(t, \cdot)$ is continuous from $[0, T]$ to $\mathcal{S}^{\prime}(\mathbb{R})$ and the right-hand side of (7.17) is continuous on [0,T] for fixed $\varphi \in \mathcal{S}(\mathbb{R})$, it is enough to show (7.17) for almost all $t \in[0, T]$.

Now for almost all $t$, the left-hand side of (7.17) is approached by the left-hand side of (7.18). Let us fix $t \in[0, T]$. It remains to show that the right-hand side of (7.17) is the limit of the right-hand side of (7.18). We fix $\omega \in \Omega$ outside a null set. We set $\mathcal{E}_{t}:=\mathcal{E}_{t}\left(\int_{0}^{\cdot} \mu\left(\mathrm{d} s, Y_{s}\right)\right), t \in[0, T]$. By Theorem 2 of [25] and uniform integrability arguments, similarly as after (2.5), we have

$$
\mathcal{E}_{t}=\exp \left(\psi_{\omega}(Y)\right)
$$

where $\psi_{\omega}: \tilde{\Omega}_{1} \rightarrow \mathbb{R}$ is a continuous modification of

$$
\omega \mapsto\left(\eta \mapsto \int_{0}^{t} e^{i}\left(\eta_{s}\right) \mathrm{d} W_{s}^{i}-\frac{1}{2} \int_{0}^{t} e^{i}\left(\eta_{s}\right)^{2} \mathrm{~d} s\right) .
$$

Indeed, previous random field, indexed by $\eta \in \tilde{\Omega}_{1}$, admits a continuous modification; to prove this we make use of Kolmogorov-Centsov theorem and Doob's inequality, which says that for any $0 \leq i \leq N$, there is a constant const $=\operatorname{const}\left(\left(e^{i}\right)^{\prime}\right)$ with

$$
E\left(\left|\int_{0}^{t}\left(e^{i}\left(\eta_{s}^{1}\right)-e^{i}\left(\eta_{s}^{2}\right)\right) \mathrm{d} W_{s}^{i}\right|^{4}\right) \leq \mathrm{const} \sup _{s \in[0, T]}\left|\eta^{1}-\eta^{2}\right|^{2}(s), \quad \eta^{1}, \eta^{2} \in \tilde{\Omega}_{1} .
$$

At this point we fix $M>0$. We decompose the difference of the right-hand sides of (7.18) and (7.17) as

$$
J_{1}(n, M)+J_{2}(n, M)+J_{3}(n, M),
$$

where

$$
\begin{aligned}
& J_{1}(n, M)=\int_{\Omega} \mathrm{d} P(\omega) R(\omega) E^{Q^{\omega, \kappa_{n}}}\left(\varphi\left(Y_{t}\right) \mathcal{E}_{t}-\varphi\left(Y_{t}\right)\left(\mathcal{E}_{t} \wedge M\right)\right), \\
& J_{2}(n, M)=\int_{\Omega} \mathrm{d} P(\omega) R(\omega)\left(E^{Q^{\omega, \kappa_{n}}}-E^{Q^{\omega}}\right)\left(\varphi\left(Y_{t}\right)\left(\mathcal{E}_{t} \wedge M\right)\right), \\
& J_{3}(n, M)=\int_{\Omega} \mathrm{d} P(\omega) R(\omega) E^{Q^{\omega}}\left(\varphi\left(Y_{t}\right)\left(\mathcal{E}_{t} \wedge M\right)-\varphi\left(Y_{t}\right) \mathcal{E}_{t}\right) .
\end{aligned}
$$

Setting $\mathbf{Q}^{\kappa_{n}}\left(\mathrm{~d} \omega, \mathrm{d} \omega_{1}\right)=\mathrm{d} P(\omega) Q^{\omega, \kappa_{n}}\left(\mathrm{~d} \omega_{1}\right)$, by Cauchy-Schwarz and Chebyshev inequalities, for every $p>1$, we have

$$
\left|J_{1}(n, M)\right|=\left|\int_{\Omega_{1} \times \Omega} \mathrm{d} Q^{\kappa_{n}} \varphi\left(Y_{t}\right) \mathcal{E}_{t} 1_{\left\{\mathcal{E}_{t}>M\right\}}\right| \leq\|\varphi\|_{\infty} \frac{E^{Q^{\kappa_{n}}}\left(\mathcal{E}_{t}^{p}\right)}{M^{p-1}} .
$$

By Lemma 7.8, we get $\sup _{n}\left|J_{1}(n, M)\right| \rightarrow 0$ if $M \rightarrow \infty$. By a similar reasoning, replacing $Q^{\kappa_{n}}\left(\mathrm{~d} \omega, \mathrm{d} \omega_{1}\right)$ with $Q\left(\mathrm{~d} \omega, \mathrm{d} \omega_{1}\right)=\mathrm{d} P(\omega) Q^{\omega}\left(\mathrm{d} \omega_{1}\right)$, we can prove that $\sup _{n}\left|J_{3}(n, M)\right| \rightarrow 0$. Let $\varepsilon>0$. Let $M$ such that 
$\sup _{n}\left|J_{1}(n, M)+J_{3}(n, M)\right| \leq \varepsilon$. On the other hand we have

$$
J_{2}(n, M)=\int_{\Omega} \mathrm{d} P(\omega) R(\omega)\left(E^{Q^{\omega, \kappa_{n}}}-E^{Q^{\omega}}\right)\left(\varphi\left(Y_{t}\right)\left(\psi_{\omega}(Y) \wedge M\right)\right) .
$$

Since for almost all $\omega, F(\eta, \omega):=R(\omega) \varphi(\eta(t)) \psi_{\omega}(\eta)$ is bounded and continuous, Corollary 7.7 implies that $J_{2}(n, M)$ goes to zero when $n \rightarrow \infty$.

Taking the limsup in (7.19) we get

$$
\limsup _{n \rightarrow \infty}\left|J_{1}(n, M)+J_{2}(n, M)+J_{3}(n, M)\right| \leq \varepsilon .
$$

Since $\varepsilon$ is arbitrarily small, we get $\lim _{n \rightarrow \infty}\left|J_{1}(n, M)+J_{2}(n, M)+J_{3}(n, M)\right|=0$ and the result follows.

\section{Appendix A: Technicalities}

Proposition A.1. Let $Y_{0}$ be distributed according to $x_{0}$. Let $a:[0, T] \times \mathbb{R} \rightarrow \mathbb{R}$ be a Borel function such there are $0<c<C$ with $c \leq a(s, \xi) \leq C, \forall(s, \xi) \in[0, T] \times \mathbb{R}$. We fix $0 \leq r \leq t \leq T$. We set $a_{n}(t, x)=\int_{\mathbb{R}} \rho_{n}(x-y) a(t, y) \mathrm{d} y$ where $\left(\rho_{n}\right)$ is the usual sequence of mollifiers converging to the Dirac delta. The unique solutions $S^{n}$ to $S_{t}^{n}=Y_{0}+$ $\int_{r}^{t} a_{n}\left(s, S_{s}^{n}\right) \mathrm{d} B_{s}, B$ being a classical Wiener process, converges in law to the (weak unique solution) of $S_{t}=Y_{0}+$ $\int_{r}^{t} a\left(s, S_{S}\right) \mathrm{d} B_{s}$.

Proof. The proof follows by standard arguments, see Stroock-Varadhan ([27], Problem 7.3.3), tightness and Kolmogorov-Centsov type arguments. For a detailed proof, the reader may consult [7].

Proof of Corollary 7.7. By (7.8), the family $\left(Q^{\kappa \ell}, \ell \in \mathbb{N}, \omega \in \Omega\right)$ is tight. So, for every positive integer $n$ there exists a compact subset $K_{n}$ of $\tilde{\Omega}_{1}$ such that

$$
Q^{\kappa_{\ell}}\left(K_{n}^{c}, \omega\right)<\frac{1}{n}, \quad \forall \ell \in \mathbb{N}, \omega \in \Omega .
$$

Since each $C\left(K_{n}\right):=C\left(K_{n} ; \mathbb{R}\right)$ is separable with respect to the sup-norm $\|\cdot\|_{\infty}$ then $C\left(K_{n}\right),\|\cdot\|_{\infty}$ is a separable Banach space. So we apply Appendix 1, Lemma A.1.4 in [21], to the map $\Omega \ni \omega \mapsto F\left(\left.\cdot\right|_{n}, \omega\right) \in C\left(K_{n}\right)$, where $F\left(\left.\cdot\right|_{K_{n}}, \omega\right)$ denotes the map $K_{n} \ni \eta \mapsto F(\eta, \omega)$. Therefore we can find a sequence $\tilde{F}_{n, k}: \Omega \rightarrow C\left(K_{n}\right)$, $\omega \mapsto \tilde{F}_{n, k}(\cdot, \omega) \in K_{n}$ such that for $\|F\|_{\infty}:=\sup _{\eta \in \tilde{\Omega}_{1}, \omega \in \Omega}|F(\eta, \omega)|$, we have

$$
\left\|\tilde{F}_{n, k}\right\|_{\infty} \leq 1+\|F\|_{\infty}, \quad \tilde{F}_{n, k}(\Omega) \subset\left\{\tilde{g}_{n, k}^{(1)}, \ldots, \tilde{g}_{n, k}^{\left(N_{n, k}\right)}\right\} \subset C\left(K_{n}\right),
$$

where $\tilde{g}_{n, k}^{(i)} \neq \tilde{g}_{n, k}^{(j)}$ if $i \neq j$, and for all $\omega \in \Omega$

$$
\sup _{\eta \in K_{n}}\left|F(\eta, \omega)-F_{n, k}(\eta, \omega)\right| \rightarrow 0
$$

as $k \rightarrow \infty$. Clearly, for all $\omega \in \Omega, \tilde{F}_{n, k}(\cdot, \omega)=\sum_{j=1}^{N_{n, k}} \tilde{g}_{n, k}^{(j)} 1_{\left\{\tilde{g}_{n, k}^{(j)}\right\}} \circ \tilde{F}_{n, k}(\cdot, \omega)$. By Tietze's extension theorem there exist extensions $g_{n, k}^{(1)}, \ldots, g_{n, k}^{\left(N_{n, k}\right)} \in C\left(\tilde{\Omega}_{1}\right)$ of $\tilde{g}_{n, k}^{(1)}, \ldots, \tilde{g}_{n, k}^{\left(N_{n, k}\right)}$ such that for all $1 \leq j \leq N_{n, k}, \sup _{\eta \in \tilde{\Omega}_{1}}\left|g_{n, k}^{(j)}(\eta)\right| \leq$ $\sup _{\eta \in \tilde{K}_{n}}\left|\tilde{g}_{n, k}^{(j)}(\eta)\right|$. Now we define $F_{n, k}: \Omega \rightarrow C\left(\tilde{\Omega}_{1}\right), \omega \mapsto F_{n, k}(\cdot, \omega)$ by

$$
F_{n, k}(\cdot, \omega)=\sum_{j=1}^{N_{n, k}} g_{n, k}^{(j)} 1_{\left\{\tilde{g}_{n, k}^{(j)}\right\}} \circ \tilde{F}_{n, k}(\cdot, \omega) .
$$


Clearly, still

$$
\left\|F_{n, k}\right\|_{\infty} \leq 1+\|F\|_{\infty} .
$$

Note that for all $\eta \in \tilde{\Omega}_{1}$

$$
\tilde{F}_{n, k}(\eta, \omega)=\sum_{j=1}^{N_{n, k}} g_{n, k}^{(j)}(\eta) 1(\eta)_{\left\{\tilde{g}_{n, k}^{(j)}\right\}} \circ \tilde{F}_{n, k}(\eta, \omega),
$$

hence of the form that Lemma 7.6 applies. Therefore using the standard notation $\mu(f):=\int f \mathrm{~d} \mu$, for a measure $\mu$ and a function $f$, we can argue as follows. Fix $n \in \mathbb{N}$. Then for all $\ell, k \in \mathbb{N}$

$$
\begin{aligned}
& \left|\int Q^{\kappa_{\ell}}(F(\cdot, \omega), \omega) P(\mathrm{~d} \omega)-\int Q(F(\cdot, \omega), \omega) P(\mathrm{~d} \omega)\right| \\
& \leq\left|\int Q^{\kappa_{\ell}}\left(F(\cdot, \omega) 1_{K_{n}}, \omega\right) P(\mathrm{~d} \omega)-\int Q\left(F(\cdot, \omega) 1_{K_{n}}, \omega\right) P(\mathrm{~d} \omega)\right|+\frac{2}{n}\|F\|_{\infty} \\
& \leq \int \underbrace{Q^{\kappa \ell}\left(\left|F(\cdot, \omega)-F_{n, k}(\cdot, \omega)\right| 1_{K_{n}}, \omega\right)}_{\leq \sup _{\eta \in K_{n}}\left|F(\eta, \omega)-F_{n, k}(\eta, \omega)\right|} P(\mathrm{~d} \omega) \\
& \quad+\left|\int Q^{\kappa_{\ell}}\left(F_{n, k}(\cdot, \omega), \omega\right) P(\mathrm{~d} \omega)-\int Q\left(F_{n, k}(\cdot, \omega), \omega\right) P(\mathrm{~d} \omega)\right| \\
& \quad+\frac{2}{n}\left(1+\|F\|_{\infty}\right)+\int Q\left(\left|F(\cdot, \omega)-F_{n, k}(\cdot, \omega)\right|, \omega\right) 1_{K_{n}} P(\mathrm{~d} \omega)+\frac{2}{n}\|F\|_{\infty} .
\end{aligned}
$$

The first inequality is a consequence of (A.1), the second one of (A.1) and (A.3). Now, letting first $\ell \rightarrow \infty$ (using Lemma 7.6), then $k \rightarrow \infty$ (using (A.2)) and finally $n \rightarrow \infty$, the assertion follows.

\section{Appendix B: Uniqueness for the porous media equation with noise}

We state here a general uniqueness lemma which only holds under even weaker hypotheses than Assumption 1.1 i.e. $\psi: \mathbb{R} \rightarrow \mathbb{R}$ is Lipschitz and that the functions belong to $W^{1, \infty}$.

Theorem B.1. Let $x_{0} \in \mathcal{S}^{\prime}(\mathbb{R})$ and suppose $\psi: \mathbb{R} \rightarrow \mathbb{R}$ to be Lipschitz. Then equation (1.1) admits at most one solution among the random fields $X:] 0, T] \times \mathbb{R} \times \Omega \rightarrow \mathbb{R}$ such that

$$
\int_{[0, T] \times \mathbb{R}} X^{2}(s, \xi) \mathrm{d} s \mathrm{~d} \xi<\infty \quad \text { a.s. }
$$

\section{Remark B.2.}

1. Suppose moreover that $e^{i}, 0 \leq i \leq N$, belong to $H^{1}$. If $x_{0} \in L^{2}$ or $\psi$ is non-degenerate then Theorem 3.4 of [8] provides an existence theorem for (1.1). It states the existence of a random field $X$ such that

$$
E\left(\int_{[0, T] \times \mathbb{R}} X^{2}(s, \xi) \mathrm{d} s \mathrm{~d} \xi\right)<\infty
$$

such that $t \mapsto X(t, \cdot)$ belongs to $C\left([0, T] ; H^{-1}(\mathbb{R})\right)$ and $t \mapsto \int_{0}^{t} \psi(X(s, \cdot)) \mathrm{d} s \in C\left([0, T] ; H^{1}(\mathbb{R})\right)$ a.s.

2. So, under the assumption of item 1., the solution $X$ is unique among those fulfilling (B.1). 
3. $X$ of point (ii) fulfills the equation, for almost all $\omega$, in $H^{-1}$

$$
X(t, \cdot)=x_{0}+\int_{0}^{t} \Delta(\psi(X(s, \cdot))) \mathrm{d} s+\int_{0}^{t} \mu(\mathrm{d} s, \cdot) X(s, \cdot), \quad t \in[0, T] .
$$

The proof of Theorem B.1 is a consequence of the result stated in Theorem 4.2 of [24], see also Theorem B.1 in [7].

\section{Acknowledgements}

The authors are grateful to the associated Editor and the two Referees for their careful reading of the first versions of the manuscript which has allowed them to considerably improve the quality of the paper. Financial support through the SFB 701 at Bielefeld University and NSF-Grant 0606615 is gratefully acknowledged. The third named author benefited partially from the support of the "FMJH Program Gaspard Monge in optimization and operation research" (Project 2014-1607H) and from the ANR Project MASTERIE 2010 BLAN 0121 01. Part of this work was written during a stay of the first and third named authors at the Bernoulli Center (EPFL Lausanne).

\section{References}

[1] V. Barbu. Analysis and Control of Nonlinear Infinite-Dimensional Systems. Mathematics in Science and Engineering. 190. Academic Press Inc., Boston, MA, 1993. MR1195128

[2] V. Barbu. Nonlinear Differential Equations of Monotone Types in Banach Spaces. Springer Monographs in Mathematics. Springer, New York, 2010. MR2582280

[3] V. Barbu, G. Da Prato and M. Röckner. Existence and uniqueness of nonnegative solutions to the stochastic porous media equation. Indiana Univ. Math. J. 57 (1) (2008) 187-211. MR2400255

[4] V. Barbu, G. Da Prato and M. Röckner. Existence of strong solutions for stochastic porous media equation under general monotonicity conditions. Ann. Probab. 37 (2) (2009) 428-452. MR2510012

[5] V. Barbu, G. Da Prato and M. Röckner. Stochastic porous media equations and self-organized criticality. Comm. Math. Phys. 285 (3) (2009) 901-923. MR2470909

[6] V. Barbu, M. Röckner and F. Russo. Probabilistic representation for solutions of an irregular porous media type equation: The degenerate case. Probab. Theory Related Fields 151 (1-2) (2011) 1-43. MR2834711

[7] V. Barbu, M. Röckner and F. Russo. A stochastic Fokker-Planck equation and double probabilistic representation for the stochastic porous media type equation. Preprint, 2014. Available at arXiv:1404.5120.

[8] V. Barbu, M. Röckner and F. Russo. Stochastic porous media equations in $\mathbb{R}^{d}$. J. Math. Pures Appl. (9) 103 (4) (2015) $1024-1052$. MR3318178

[9] N. Belaribi, F. Cuvelier and F. Russo. A probabilistic algorithm approximating solutions of a singular pde of porous media type. Monte Carlo Methods Appl. 17 (4) (2011) 317-369. MR2890425

[10] N. Belaribi and F. Russo. Uniqueness for Fokker-Planck equations with measurable coefficients and applications to the fast diffusion equation. Electron. J. Probab. 17 (84) (2012) 28. MR2988399

[11] S. Benachour, P. Chassaing, B. Roynette and P. Vallois. Processus associés à l'équation des milieux poreux. Ann. Scuola Norm. Sup. Pisa Cl. Sci. (4) 23 (4) (1996) 793-832. MR1469575

[12] P. Benilan, H. Brezis and M. G. Crandall. A semilinear equation in $L^{1}\left(\mathbb{R}^{N}\right)$. Ann. Scuola Norm. Sup. Pisa Cl. Sci. (4) 2 (4) (1975) 523-555. MR0390473

[13] P. Benilan and M. G. Crandall. The continuous dependence on $\varphi$ of solutions of $u_{t}-\Delta \varphi(u)=0$. Indiana Univ. Math. J. 30 (2) (1981) 161-177. MR0604277

[14] P. Blanchard, M. Röckner and F. Russo. Probabilistic representation for solutions of a porous media type equation. Ann. Probab. 38 (5) (2010) 1870-1900. MR2722788

[15] H. Brezis and M. G. Crandall. Uniqueness of solutions of the initial-value problem for $u_{t}-\Delta \varphi(u)=0$. J. Math. Pures Appl. (9) 58 (2) (1979) 153-163. MR0539218

[16] E. Häusler and H. Luschgy. Stable Convergence and Stable Limit Theorems. Probability Theory and Stochastic Modelling 74. Springer, Cham, 2015. MR3362567

[17] J. Jacod and A. N. Shiryaev. Limit Theorems for Stochastic Processes, 2nd edition. Grundlehren der Mathematischen Wissenschaften [Fundamental Principles of Mathematical Sciences] 288. Springer-Verlag, Berlin, 2003. MR1943877

[18] I. Karatzas and S. E. Shreve. Brownian Motion and Stochastic Calculus, 2nd edition. Graduate Texts in Mathematics 113. Springer-Verlag, New York, 1991. MR1121940

[19] H. P. McKean Jr. Propagation of chaos for a class of non-linear parabolic equations. In Stochastic Differential Equations (Lecture Series in Differential Equations, Session 7, Catholic Univ., 1967) 41-57. Air Force Office Sci. Res., Arlington, Va, 1967. MR0233437 
[20] É. Pardoux. Filtrage non linéaire et équations aux dérivées partielles stochastiques associées. In École d'Été de Probabilités de Saint-Flour XIX-1989 67-163. Lecture Notes in Math. 1464. Springer, Berlin, 1991. MR1108184

[21] C. Prévôt and M. Röckner. A Concise Course on Stochastic Partial Differential Equations. Lecture Notes in Mathematics 1905. Springer, Berlin, 2007. MR2329435

[22] J. Ren, M. Röckner and F.-Y. Wang. Stochastic generalized porous media and fast diffusion equations. J. Differential Equations 238 (1) (2007) 118-152. MR2334594

[23] B. D. Ripley. The disintegration of invariant measures. Math. Proc. Cambridge Philos. Soc. 79 (2) (1976) 337-341. MR0404581

[24] Röckner and F. Russo. Uniqueness for stochastic Fokker Planck and porous media equations in the sense of distributions. J. Evol. Equ. To appear. Available at http://arxiv.org/abs/1609.00165. MR3707309

[25] F. Russo and P. Vallois. Elements of stochastic calculus via regularization. In Séminaire de Probabilités XL 147-185. Lecture Notes in Math. 1899. Springer, Berlin, 2007. MR2409004

[26] R. E. Showalter. Monotone Operators in Banach Space and Nonlinear Partial Differential Equations. Mathematical Surveys and Monographs 49. American Mathematical Society, Providence, RI, 1997. MR 1422252

[27] D. W. Stroock and S. R. S. Varadhan. Multidimensional Diffusion Processes. Classics in Mathematics. Springer-Verlag, Berlin, 2006. Reprint of the 1997 edition. MR2190038

[28] J.-L. Vázquez. The Porous Medium Equation. Oxford Mathematical Monographs. Mathematical Theory. The Clarendon Press Oxford University Press, Oxford, 2007. MR2286292 